\title{
THREE-DIMENSIONAL SIMULATIONS OF MHD JET PROPAGATION THROUGH UNIFORM AND STRATIFIED EXTERNAL ENVIRONMENTS
}

\author{
S. M. O’Neill, ${ }^{1}$ I. L. Tregillis, ${ }^{2}$ T. W. Jones, ${ }^{1}$ And Dongsu Ryu ${ }^{3}$ \\ Received 2005 June 15; accepted 2005 July 25
}

\begin{abstract}
We present a set of high-resolution three-dimensional MHD simulations of steady light, supersonic jets, exploring the influence of jet Mach number and the ambient medium on jet propagation and energy deposition over long distances. The results are compared to simple self-similar scaling relations for the morphological evolution of jet-driven structures and to previously published two-dimensional simulations. For this study we simulated the propagation of light jets with internal Mach numbers 3 and 12 to lengths exceeding 100 initial jet radii in both uniform and stratified atmospheres. The propagating jets asymptotically deposit approximately half of their energy flux as thermal energy in the ambient atmosphere, almost independent of jet Mach number or the external density gradient. Nearly one-quarter of the jet total energy flux goes directly into dissipative heating of the ICM, supporting arguments for effective feedback from AGNs to cluster media. The remaining energy resides primarily in the jet and cocoon structures. Despite having different shock distributions and magnetic field features, global trends in energy flow are similar among the different models. As expected, the jets advance more rapidly through stratified atmospheres than uniform environments. The asymptotic head velocity in King-type atmospheres shows little or no deceleration. This contrasts with jets in uniform media with heads that slow as they propagate. This suggests that the energy deposited by jets of a given length and power depends strongly on the structure of the ambient medium. While our low Mach number jets are more easily disrupted, their cocoons obey evolutionary scaling relations similar to the high Mach number jets.
\end{abstract}

Subject headings: galaxies: jets - methods: numerical — MHD

Online material: color figures, mpeg animations

\section{INTRODUCTION}

The important roles of supersonic jets in various astrophysical contexts, such as radio galaxies, are now well established. Still, supersonic jet propagation and interaction with the ambient medium are complex, and remain topics of current research interest. Studies of fully three-dimensional flows are quite limited, especially for jet propagation over distances having length-toradius ratios comparable to those observed. Among several dynamical issues, two have particular general importance, namely, the character of energy transfer from active jets to their environments and jet length and flow morphology evolution in time.

Energetic jets are commonly invoked as mechanisms responsible for disrupting cooling flows and heating cluster environments (e.g., Böhringer et al. 2002; Churazov et al. 2003; Blanton et al. 2003; Zanni et al. 2005). Once a jet ceases to be powered at its source, the energy in its cocoon may remain inside a buoyant bubble that does mostly adiabatic work on the environment until it is disrupted (e.g., Brüggen et al. 2002; Fabian et al. 2003; Robinson et al. 2004; Jones \& DeYoung 2005). At that time, the remaining bubble energy that has not been radiated or conducted away is shared. Two of the more immediate, critical questions concerning jets are how much of the energy flux carried by an active jet is shared with the environment and how much of that appears as irreversible heat.

\footnotetext{
1 School of Physics and Astronomy, University of Minnesota, Minneapolis, MN 55455; smoneil@msi.umn.edu,twj@msi.umn.edu.

2 Applied Physics Division, MS B259, Los Alamos National Laboratory, Los Alamos, NM 87545; iant@lanl.gov.

3 Department of Astronomy and Space Science, Chungnam National University, Daejeon 305-764, South Korea; ryu@canopus.cnu.ac.kr.
}

Models of the evolution of flow morphology provide further insight into energy deposition by active jets. Analytic models, such as those developed by Cioffi \& Blondin (1992), Kaiser \& Alexander (1997), Komissarov \& Falle (1998), and Alexander (2000), have been used to describe the expected morphology evolution of these systems in an attempt to understand their distribution in several observational planes. Such simple self-similar relations allow for the estimation of energy densities in these systems independent of detailed source histories. Complimentary two-dimensional numerical simulations of jets, such as those done by Norman et al. (1982), Cox et al. (1991), Falle (1991), Cioffi \& Blondin (1992), Hardee et al. (1992), Komissarov \& Falle (1997), and Carvalho \& O'Dea (2002a, 2002b), have been conducted to examine the validity of these simple models. Simulations have illustrated the complexity of jet flows and have helped immensely in exploring the physical and environmental parameter space available to these objects. Furthermore, Zanni et al. (2005) recently described a set of two-dimensional hydrodynamical simulations of jets propagating into realistic cluster environments.

Still, fully three-dimensional simulations provide the best approach to addressing the issues raised above, since such simulations can follow the highly complex flows driven by jets and allow for a full accounting of the energy transport in these systems. Previous three-dimensional simulations, such as those conducted by Hardee \& Clarke (1992), Hooda \& Wiita (1996), and Norman (1996), have successfully illustrated flow features unique to three dimensions, the latter having simulated a threedimensional jet over 100 jet radii in length. Krause $(2003,2005)$ has further explored the propagation of two-dimensional axisymmetric and fully three-dimensional evolved jets into uniform and King atmospheres, examining the shape of the bow shock 
TABLE 1

Summary of Simulations

\begin{tabular}{|c|c|c|c|c|c|c|c|}
\hline $\mathrm{ID}^{\mathrm{a}}$ & $M^{\mathrm{b}}$ & $M_{j}$ & Atmosphere & $\begin{array}{c}x_{\text {size }} \\
(\mathrm{kpc})\end{array}$ & $\begin{array}{c}y, z_{\text {size }}{ }^{\mathrm{c}} \\
(\mathrm{kpc})\end{array}$ & $\begin{array}{c}\text { Final Age } \\
\text { (Myr) }\end{array}$ & $\begin{array}{c}L_{j}{ }^{\mathrm{d}} \\
\left(\operatorname{ergs~s}^{-1}\right)\end{array}$ \\
\hline HU............. & 120 & 12 & Uniform (U) & 230 & 228 & $\approx 52$ & $1.67 \times 10^{46}$ \\
\hline HU-r ......... & 120 & 12 & Uniform (U) & 230 & 633 & $\approx 52$ & $1.67 \times 10^{46}$ \\
\hline HK.......... & 120 & 12 & King-type (K) & 230 & 228 & $\approx 26$ & $1.67 \times 10^{46}$ \\
\hline LU ............... & 30 & 3 & Uniform (U) & 230 & 903 & $\approx 66$ & $1.53 \times 10^{45}$ \\
\hline 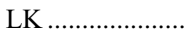 & 30 & 3 & King-type (K) & 230 & 633 & $\approx 37$ & $1.53 \times 10^{45}$ \\
\hline
\end{tabular}

\footnotetext{
a HU-r model features reflecting boundaries surrounding a $x=0$ jet orifice. All other models have open boundaries everywhere.

b Jet speed are fixed at $v_{j}=0.15 c$. Mach number are adjusted with ICM density and sound speed.

c Grid size are designed always to include bow wave. The outer zones in $y$ and $z$ dimensions are never disturbed, and the total grid size in some cases greatly exceeds the size of the disturbed regions.

d Jet densities are calculated from fixed $\rho_{j}=\eta \rho_{0}$, with $\eta=0.01$. ICM base density is given by $\rho_{0}=1.18 \times$

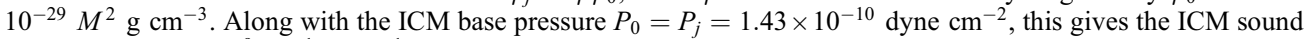
speed $c_{a}=4.50 \times 10^{9} M^{-1} \mathrm{~cm} \mathrm{~s}^{-1}$. Jet magnetic fields include a poloidal component equal to the ambient field $\beta=100$ at $x=0$ as well as a toroidal component whose peak value is twice the poloidal value.
}

structures over time and exploring the influence of boundary conditions on the propagation of jet-driven structures.

Our previous work, described in Tregillis et al. (2001a, 2001b, 2004), illustrated the complexity of the shock and magnetic field structures generated in full three-dimensional jetdriven flows and described how detailed information about nonthermal particle populations in these systems is essential to correctly relate observations to the antecedent physical structures. In the present work, we examine the long-term evolution of steady light three-dimensional magnetohydrodynamic (MHD) jets, exploring how the energetics, dynamics, and morphology of the bulk plasma evolve, and whether they do so in a simple manner. To assure that fully three-dimensional dynamics are as divorced as possible from start-up behaviors, we follow the evolution of each jet and its neighboring environment until the jets have penetrated more than 100 initial jet radii into those environments. We model both high and low Mach number jet behaviors and also consider uniform and stratified ambient media. We examine for each system the time evolution of energy flow, computing the amount of inflowing energy deposited in the environment and determining how energy is partitioned within the disturbed flows. We further observe how jet length and flow morphology evolve in time and compare our threedimensional results to those of two-dimensional simulations and models to illustrate which features of jet-driven flows are well described by simple models and which features differ in detailed treatments.

In $\S 2$, we discuss the details of our numerical methods and simulation properties. The analysis of our simulated data is described in $\S 3$, and conclusions and astrophysical implications are discussed in $\S 4$.

\section{CALCULATION DETAILS}

\subsection{Numerical Methods}

Our simulations are carried out on a three-dimensional Cartesian grid. They employ a second-order total variation diminishing (TVD) nonrelativistic ideal MHD code, as described in Ryu \& Jones (1995) and Ryu et al. (1998). The method conserves mass, momentum, and energy to machine accuracy. To set up hydrostatic equilibrium in the ambient media, external gravity is added as a source term in the $x$-direction through operator splitting, applying the $x$-momentum correction at each time step to recalculate the total energy. In this treatment of gravity, mo- mentum and energy are no longer exactly conserved. However, we have confirmed that associated errors are much too small to influence our results. The code maintains a divergence-free magnetic field at each time step using a constrained transport scheme (Ryu et al. 1998). A gamma-law gas equation of state is assumed, with $\gamma=5 / 3$.

A passive mass fraction or "color" tracer, $C_{j}$, is introduced at the jet orifice to track jet material as it propagates through the computational grid. The value of $C_{j}$ is set to unity in the jet, while $C_{j}=0$ in the ambient medium. Passive nonthermal, relativistic electrons are included as well (see, e.g., Jones et al. 1999; Tregillis et al. 2001a, 2004) in order to model nonthermal emissions from the flows. We restrict our present analysis to the bulk flow, leaving the complimentary emission analysis to a separate paper (S. M. O'Neill et al., in preparation).

Our simulated jets propagate approximately along the $x$-axis after entering the grid through a circular orifice centered in the $x=x_{0}=0$ plane. The computational box extends to $x=x_{1}=$ $230 \mathrm{kpc}$, spanned by a grid of 576 uniform zones $(\Delta x \approx 0.4 \mathrm{kpc})$. The equal, transverse $y$ - and $z$-dimensions of the box are selected for each simulation so that they contain the entire jet bow wave until the end of the simulation. Within $25 \mathrm{kpc}$ of the box midline, the transverse grid zones are uniform $(\Delta y=\Delta z=\Delta x)$. Exterior $y$ and $z$ zone sizes expand logarithmically with an expansion factor 1.1 , out to a maximum zone size of $8.4 \mathrm{kpc}$. Box dimensions for each simulation are listed in Table 1.

Continuous boundary conditions are employed for both extremes of $y$ and $z$. A modified continuous condition is applied at $x_{1}$, designed to maintain hydrostatic equilibrium in the undisturbed medium. Inflow boundaries are applied inside the jet orifice on the $x_{0}$ boundary. With the exception of one simulation (HU-r, described below), the same modified continuous boundary condition is applied on the rest of the $x_{0}$ plane as on the $x_{1}$ plane. In the HU-r simulation, reflecting boundaries are applied at $x_{0}$ outside the jet orifice.

\subsection{Simulation Properties}

We discuss five simulations, including Mach 12 jets (labeled " $\mathrm{H}$ " for "high Mach number") and Mach 3 jets (labeled "L" for "low Mach number"). For each Mach number we simulate jets penetrating uniform media (labeled " $U$ ") and stratified, King-type media (labeled " $\mathrm{K}$ "). Except for the entrance plane of the jet $(x=0)$, all boundaries remain undisturbed during all the simulations. This feature is necessary for us to examine the 


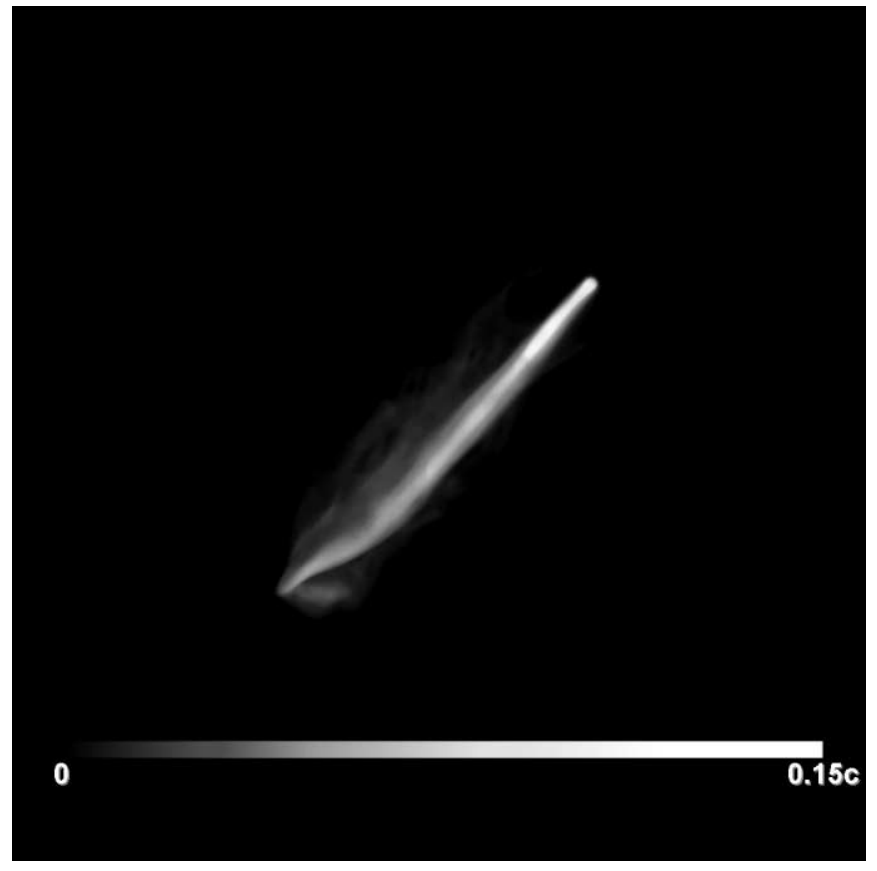

FIG. 1.-Volume rendering of flow speed for the HU-r model, after the jet has propagated $\sim 90 \%$ of the total grid length. [See the electronic edition of the Journal for a color version of this figure and an animation of this quantity as seen from several different angles.]

influence of the jet on the ambient medium and especially on its energetics. It is not possible to avoid influences from the jet inflow plane, so long as that is a grid boundary. To evaluate the role of this boundary, we computed a pair of "HU" simulations, one with open (continuous) boundaries on this plane and one with reflecting boundaries (leading to the "HU-r" label). We briefly outline the properties of the jets and the ambient media in the following two subsections. The physical parameters of each simulation are summarized in Table 1.

\subsubsection{The Jets}

The jet inflows are steady after a brief starting sequence. The incoming jet flow slowly wobbles in a $3^{\circ}$ cone around the $x$-direction. This establishes fully three-dimensional flows within the physical domain as early as possible. Our five model jets are identical except for Mach number and period of the induced jet wobble. The entering jets have uniform cores of radius $r_{j}=2 \mathrm{kpc}$ surrounded by a concentric transition annulus that smoothly connects to the ambient conditions. The jet core speed is $v_{j}=$ $0.15 c$. The core density is $\rho_{j}=\eta \rho_{0}$, where $\rho_{0}$ is the ambient density at $x=0$ (discussed below), and we set $\eta=0.01$ in each simulation. The jets enter with the same gas pressure as the local ambient medium, namely $P_{0}$, as discussed below. Earlier twodimensional studies have pointed to significant dynamical dependencies on jet Mach number (e.g., Carvalho \& O'Dea 2002a, 2002 b). Our jets are parameterized by internal Mach numbers, $M_{j}$, making the internal jet sound speed $c_{j}=v_{j} / M_{j}$. We discuss simulations applying $M_{j}=12$ and $M_{j}=3$. As noted before, we designate the former as high Mach number $(\mathrm{H})$ and the latter as low Mach number (L) jets. The period of the inflowing jet wobble is $16 \mathrm{Myr}$ for the $\mathrm{H}$ model jets and $4 \mathrm{Myr}$ for the L model jets.

The inflowing jet power is calculated from $L_{j} \approx L_{k}+L_{t}$, where $k$ and $t$ refer to the kinetic and thermal components, respectively, ignoring the negligible contributions of magnetic and gravitational energy to the total power. The power

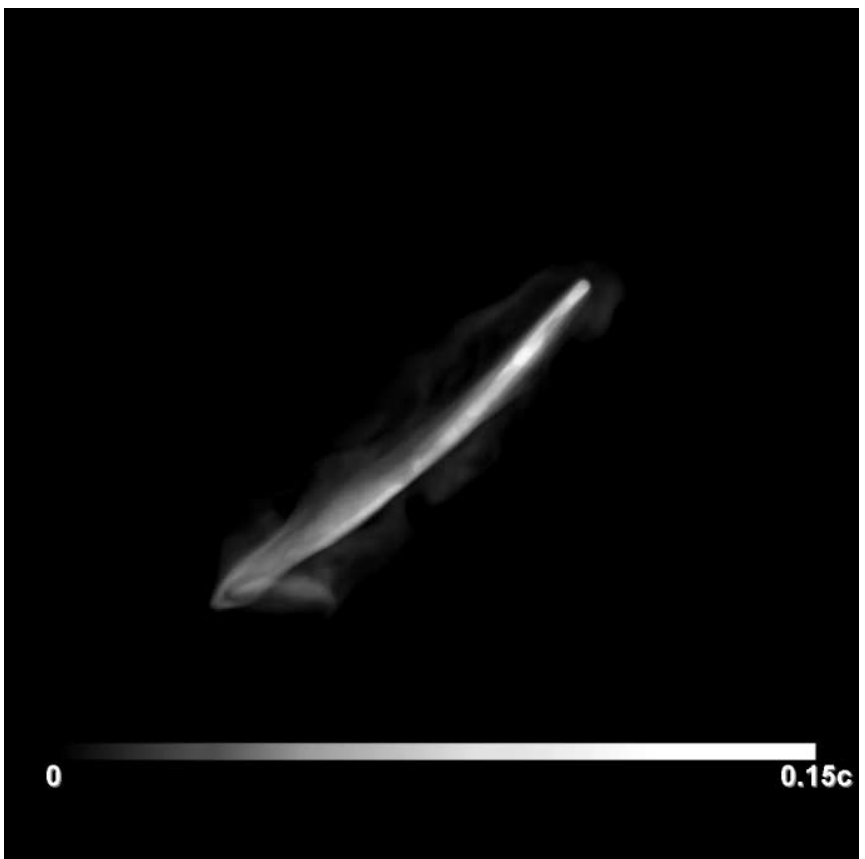

FIG. 2.-As in Fig. 1, but for the HU model. [See the electronic edition of the Journal for a color version of this figure and an animation of this quantity as seen from several different angles.]

components are given by $L_{k}=\int\left(\frac{1}{2} \rho_{j} v^{2}\right) v_{x} d A$ and $L_{t}=\int[\gamma(\gamma-$ $\left.1)^{-1} P_{j}\right] v_{x} d A$, where the integrals span the core and transition inflow regions. Inserting physical values (see Table 1) and ignoring discretization on the grid, this allows us to compute a nominal jet power simply as a function of jet Mach number, namely,

$$
L_{j} \approx 1.12 \times 10^{44}\left(M_{j}^{2}+4.6\right) \mathrm{erg} \mathrm{s}^{-1} .
$$

The actual, measured energy flow onto the grid is slightly less than this estimate due to grid discretization and back-reaction of the flows on the grid near the perimeter of the jet orifice. The difference is asymptotically only about $1 \%$, so this analytic expression provides a very good measure of the energy added to the volumes being modeled (see $\S 3.1$ ). To avoid start-up difficulties, the jet speeds were ramped to full value over a finite time that depended on the Mach number. Jet penetration and energy deposition during that time were negligible, so in the discussion below we reset the simulation clocks to start at the time when the jets reach full speed.

The jet magnetic field consists of toroidal and poloidal components. The inflowing jet poloidal field is equal to the uniform ambient field, $B_{x 0}$, discussed below. The toroidal field inside the jet core is $B_{\phi 0}=1.25 B_{x 0}\left(r / r_{j}\right)$. This field component decreases quadratically to zero across the transition annulus.

Figures 1-5 show volume renderings of flow speed at late times for each of the five models. Viewing these images and the associated animations available in the electronic version of this paper provides an efficient introduction to the behaviors of each simulation and their intercomparison. In each figure, the high-velocity jet enters the grid from the upper right, inflating a cocoon of material that has entered the grid through the jet orifice; the animations show the time evolution of these structures from several viewing angles. Propagation times across the grid vary with Mach number and especially with the structure of the ambient medium. They range from 26 to $66 \mathrm{Myr}$, as listed in Table 1. 


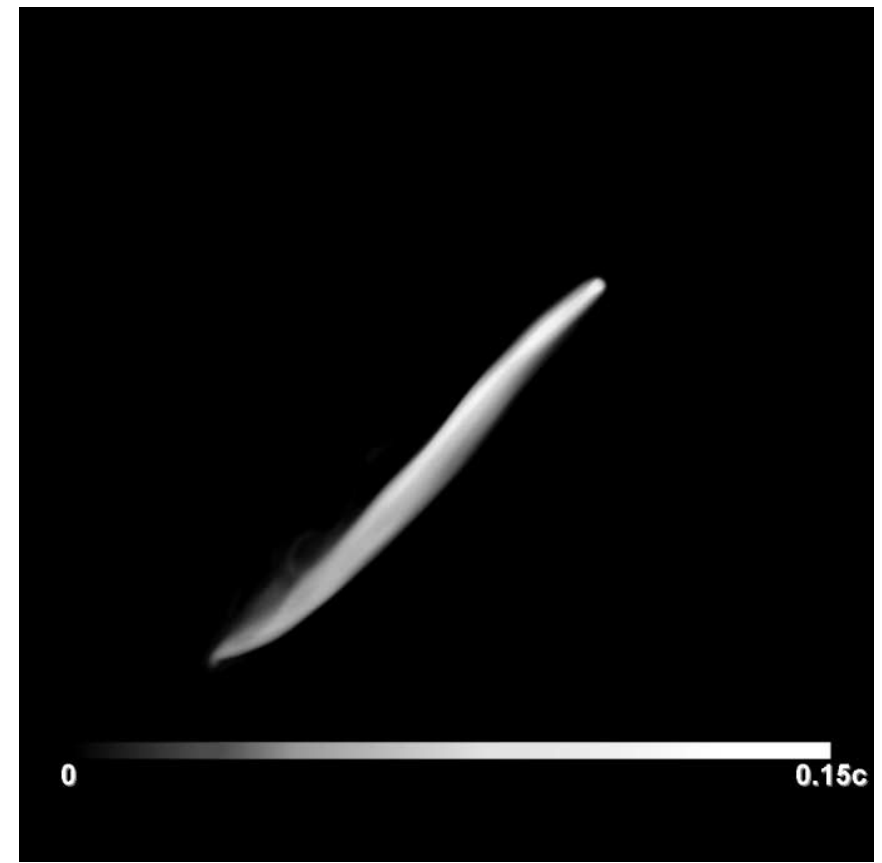

FIG. 3.-As in Fig. 1, but for the HK model. [See the electronic edition of the Journal for a color version of this figure and an animation of this quantity as seen from several different angles.]

\subsubsection{The Ambient Medium}

We consider two simple model equilibrium atmospheres: a uniform (U) and a plane-stratified medium. The stratified ICM is a simple, isothermal King-type form (King 1962) (K; see Fig. 6),

$$
\rho_{a}(x)=\frac{\rho_{0}}{1+\left(x / x_{c}\right)^{2}},
$$

where $\rho_{0}$ is the density at $x_{0}$. For the $\mathrm{K}$ model atmosphere, $x_{c}=\frac{1}{3} x_{1} \approx 76.7 \mathrm{kpc}$. The $\mathrm{U}$ model atmosphere corresponds to

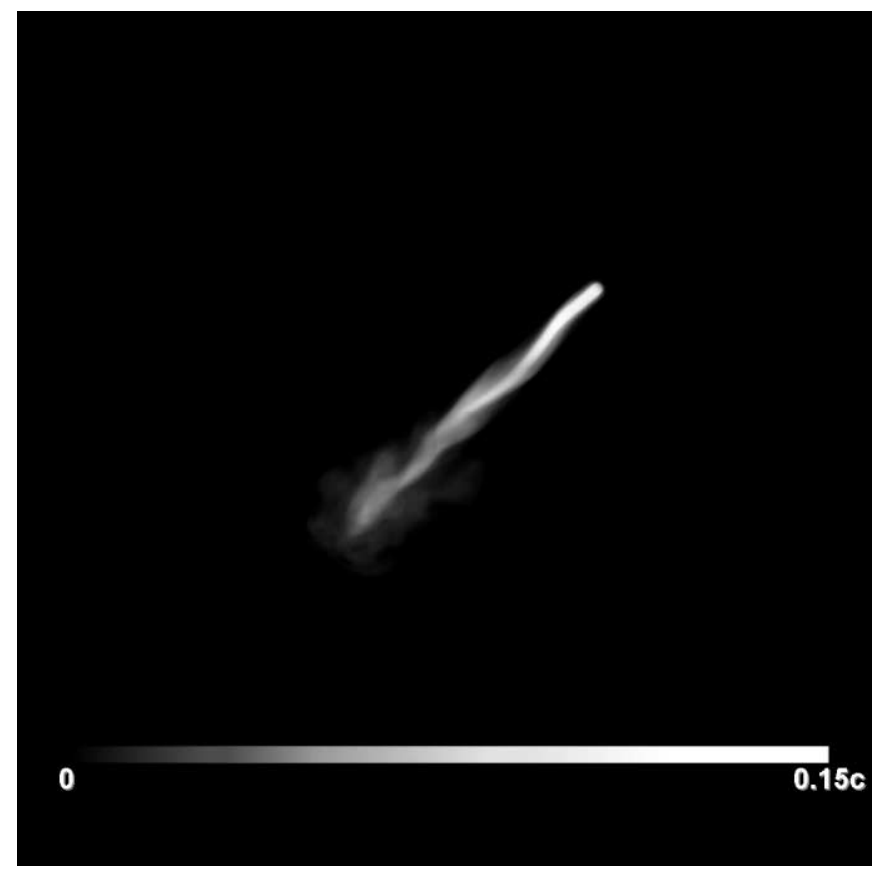

FIG. 4.-As in Fig. 1, but for the LU model. [See the electronic edition of the Journal for a color version of this figure and an animation of this quantity as seen from several different angles.]

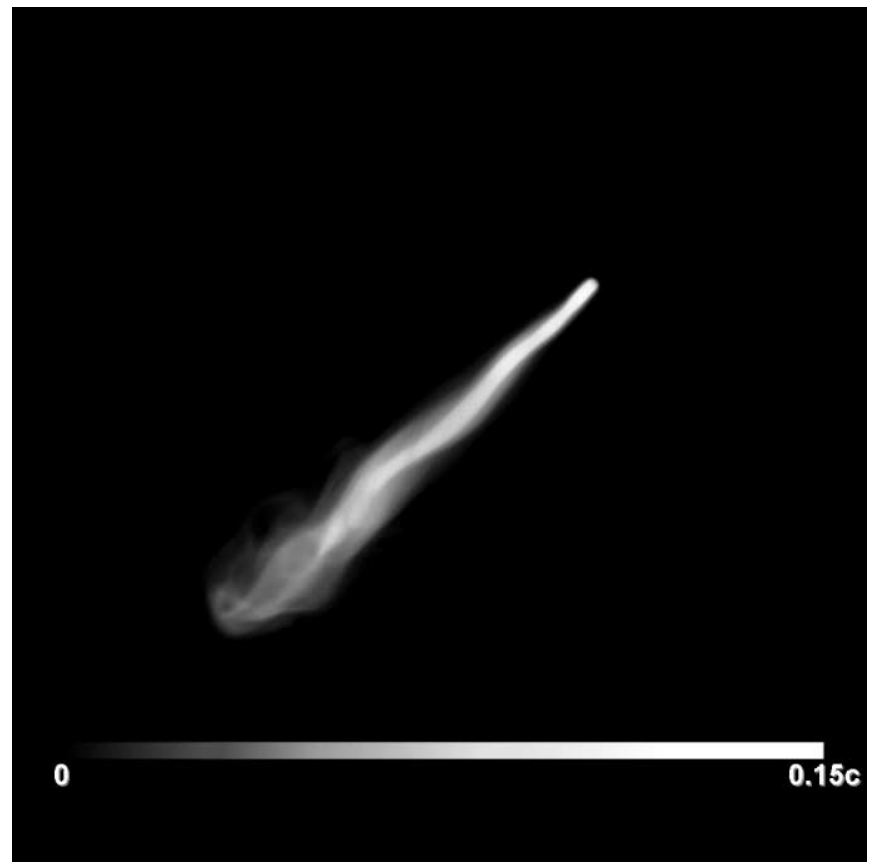

FIG. 5.-As in Fig. 1, but for the LK model. [See the electronic edition of the Journal for a color version of this figure and an animation of this quantity as seen from several different angles.]

$x_{c} \rightarrow \infty$. The initial ICM pressure is simply $P(x)=c_{a}^{2}\left[\rho_{a}(x) / \gamma\right]=$ $P_{0}\left[\rho_{a}(x) / \rho_{0}\right]$, where $c_{a}$ is the ambient sound speed, and for all simulations $P_{0}=1.43 \times 10^{-10}$ dyne $\mathrm{cm}^{-2}$. The ambient sound speed is set by the jet Mach number from the jet-ICM density contrast, $\eta$, and the assumption of jet-ICM pressure balance, namely, $c_{a}=v_{j} \sqrt{\eta} / M_{j}$. Accordingly, the core jet speed has a Mach number with respect to $c_{a}$ given by $M=M_{j} / \sqrt{\eta}$. For our jet parameters, the associated ICM temperatures for the $M_{j}=$ 12 (3) jets are 0.88 (14) $\mu \mathrm{keV}$, where $\mu$ is the mean molecular weight of the ICM. These parameters are chosen such that the

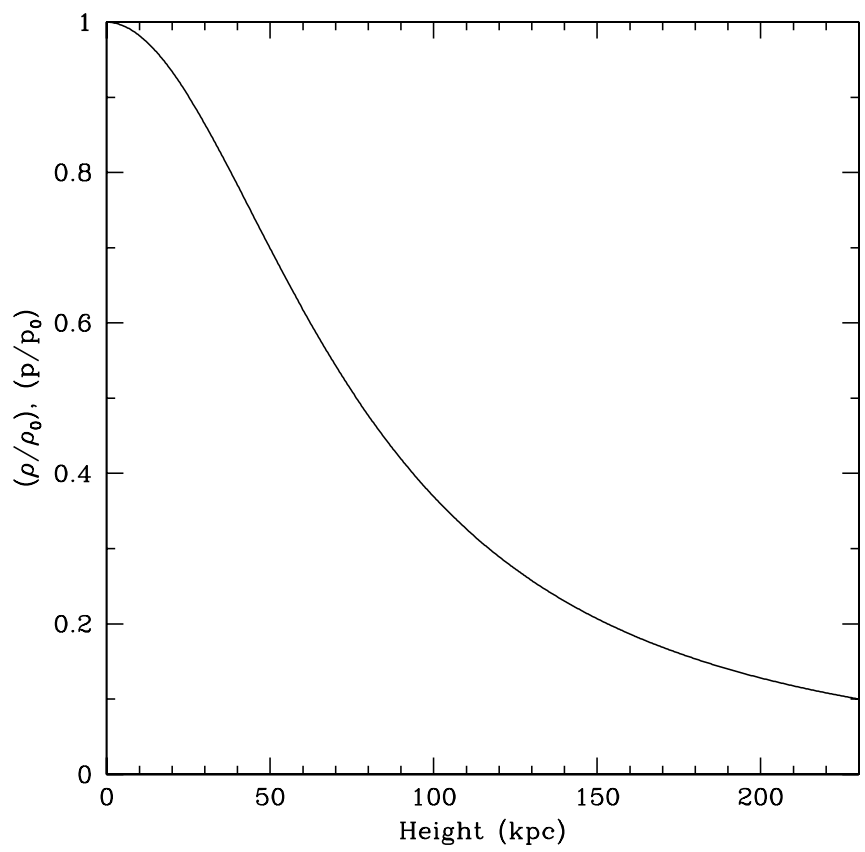

FIG. 6.- Initial King-type density and pressure profiles, used in the HK and LK models. Values of $\rho_{0}$ and $P_{0}$ are given in Table 1. 
$\mathrm{H}$ models include temperatures appropriate for cooling-flow clusters with cooled cores, while the L models describe hotter massive clusters. The base ICM density is then $\rho_{0}=\gamma P_{0} / c_{a}^{2}=$ $1.18 \times 10^{-29} \mathrm{M}^{2} \mathrm{~g} \mathrm{~cm}^{-3}$. The inferred gravitational acceleration is

$$
g(x)=-\frac{2 c_{a}^{2}}{\gamma} \frac{x}{x_{c}^{2}+x^{2}},
$$

which vanishes in the $\mathrm{U}$ models. This gravity model is not truly representative of those in real clusters. Its only purpose here is to allow establishment of hydrostatic equilibrium in the undisturbed stratified medium. Otherwise gravity plays a negligible role in these simulations. The initial ambient magnetic field in each simulation is uniform and in the $x$-direction, i.e., $B_{x}=B_{x_{0}}=3 \mu \mathrm{G}$. The resulting magnetic pressure at $x_{0}$ is $1 \%$ of the gas pressure; i.e., $\beta_{0}=P_{g} / P_{B}=100$. At the top of the King atmosphere, $\beta=10$. This value of $B_{x 0}$ is in the range of values suggested by observations of cluster media. The specific value was selected to control the synchrotron loss times of the relativistic electrons transported in the simulated flows. While that issue is not included in the present discussion, it will be important to our subsequent discussion of the radiant luminosity evolution of the simulated objects (S. M. O'Neill et al., in preparation).

\section{DISCUSSION}

\subsection{Energy Flows}

By isolating contributions from jet plasma and the ICM using the "jet color" tracer, we can characterize where energy in the system is transported and how much of it goes toward direct heating of the ambient medium. Quantitatively following the flow of kinetic, thermal, magnetic, and gravitational energy in each simulation further allows us to characterize how energy in the system changes form. We begin our analysis by examining how energy brought onto the grid by the jet is exchanged with the ambient medium and how it becomes partitioned among different energy forms as a result of the complex dynamics of the jet-ICM interaction. The generation of thermal energy in the ICM and especially dissipated heat is of special significance. We also examine how this energy flow is affected by the structure of the ambient medium and jet Mach number. Since the final propagation lengths of all our jets are the same, but the propagation times span a broad range, it is most convenient to present many of our results in terms of jet length rather than time. In the following subsection we explore the dynamical time evolution of each jet, including its length evolution, so that one can translate length behaviors into time behaviors, if desired (see $\S 3.2$ and the upper two panels of Fig. 18). In these discussions we define the length of the jet as the largest value of $x$ contained by the bow shock preceding the jet. Typically the jet (beam) terminus and the extremum of the bow shock are at almost the same location. That position also defines the "head" of the jet, $x_{h}$, so the velocity of the jet head refers to $d x_{h} / d t$. We note, however, that the head of the jet is not generally on the axis defined by the jet orifice, due to the jet wobble and especially the sometimes dramatic dynamical instabilities experienced by the jet tip.

The bottom right panels of Figures 7-11 provide a basic accounting of the energy changes introduced on the grid in each simulation. In each case the solid line represents an integration of the jet power, given approximately by equation (1), while the dotted lines represent the measured total change in energy since the simulation began. Figure 7 illustrates the result for the HU-r model, where no energy is allowed to leave the grid. Ideally we would expect the two curves to overlap in this case. As noted above, they agree well, but not exactly, the measured increase being slightly smaller. The difference comes from the backreaction of the jet cocoon on the jet perimeter near the orifice. That effect diminishes with time, so that asymptotically, the nominal and actual energy fluxes agree to within about $1 \%$.

All the other simulations utilize open or continuous boundaries along the jet inflow plane. As illustrated in Figures 8-11, and as one would expect, the measured energy changes in those cases are reduced by outflows across this plane. Still, those losses are quite modest, being asymptotically $\$ 13 \%$ for all models. We defer to $\S 3.2$ a discussion of the dynamical influences of $x=0$ boundary conditions.

Having established a reasonable accounting of the global energy changes in the different simulations, we next examine the energy transferred to the ambient medium from the jet penetrating it. To do that, we isolate the jet and its cocoon using the color tracer, $C_{j}$. Figure 12 shows, as a function of jet length, the fractional change in kinetic and thermal energy in the ambient medium compared to the total, measured energy added to the grid. These fractions are found by integrating each energy form over the computational grid, weighted by the passive color tracer (actually $1-C_{j}$ ). We note that this result differs by at most a few percent from that obtained by isolating and removing the jet/jet cocoon using a color threshold, such as $C_{j}=0.9$. Thus, relatively little energy has been exchanged by entrainment (indicated by intermediate values of $C_{j}$ ) during the periods simulated. The plots show that in each of the $\mathrm{H}$ models, approximately $55 \%-60 \%$ of the jet energy is transferred to the ambient medium; while about $45 \%$ of the $\mathrm{L}$ model jet energies are given to the ambient medium. Similarly, approximately $40 \%-45 \%$ of the jet energy is converted in each case to ambient thermal energy. Those transfer fractions, and especially the total energy measures, are roughly constant once each jet has penetrated more than about 50 jet radii into their environments. Thus, these figures seem to represent fair estimates of the steady, asymptotic energy exchange rates between such jets and their surroundings. It is remarkable that they depend only weakly on the Mach number of the jet or on the density profile of the ambient medium. In addition, we estimate that between $40 \%-60 \%$ of the thermal energy entering the ambient medium is added irreversibly, mostly through shock dissipation. The ambient irreversible (entropy-enhancing) energy change estimate is obtained by subtracting adiabatic changes due to compression, given by $\Delta E_{\text {ad }}=-\int d V \int \gamma(\gamma-1)^{-1} P(\nabla \cdot v) d t$, from the measured change in ambient thermal energy. Thus, a net $\sim 20 \%-30 \%$ of the steady jet power goes directly into entropy generation within the ICM. The efficient transfer of thermal energy from our simulated jets to their environments is significant, because it gauges the potential for active galaxies to provide energetic feedback to cluster environments while their jets are "on."

Zanni et al. (2005) have recently examined this same question based on two-dimensional, axisymmetric jet simulations. Their jets were switched off relatively early, before they had penetrated more than about 20 jet radii into their environments. So, close comparison of our results with theirs is difficult. However, from their Figure 8 we can estimate that in each of several simulations about $15 \%$ of their jet energy was dissipated irreversibly at the time the jet was switched off. That is very consistent with our findings. Their simulated jet parameters extended to higher Mach numbers and larger density contrasts 

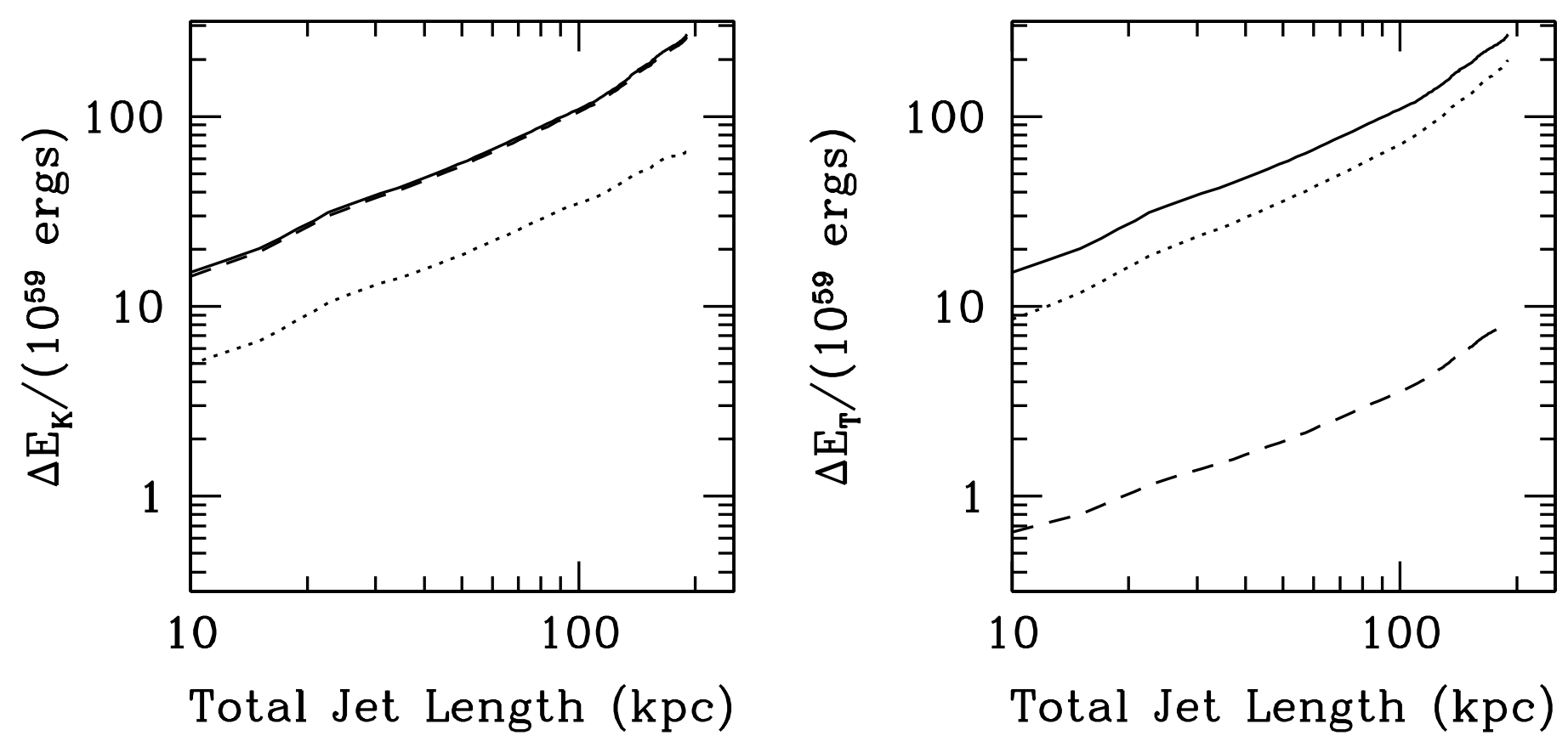

Total Jet Length (kpc)
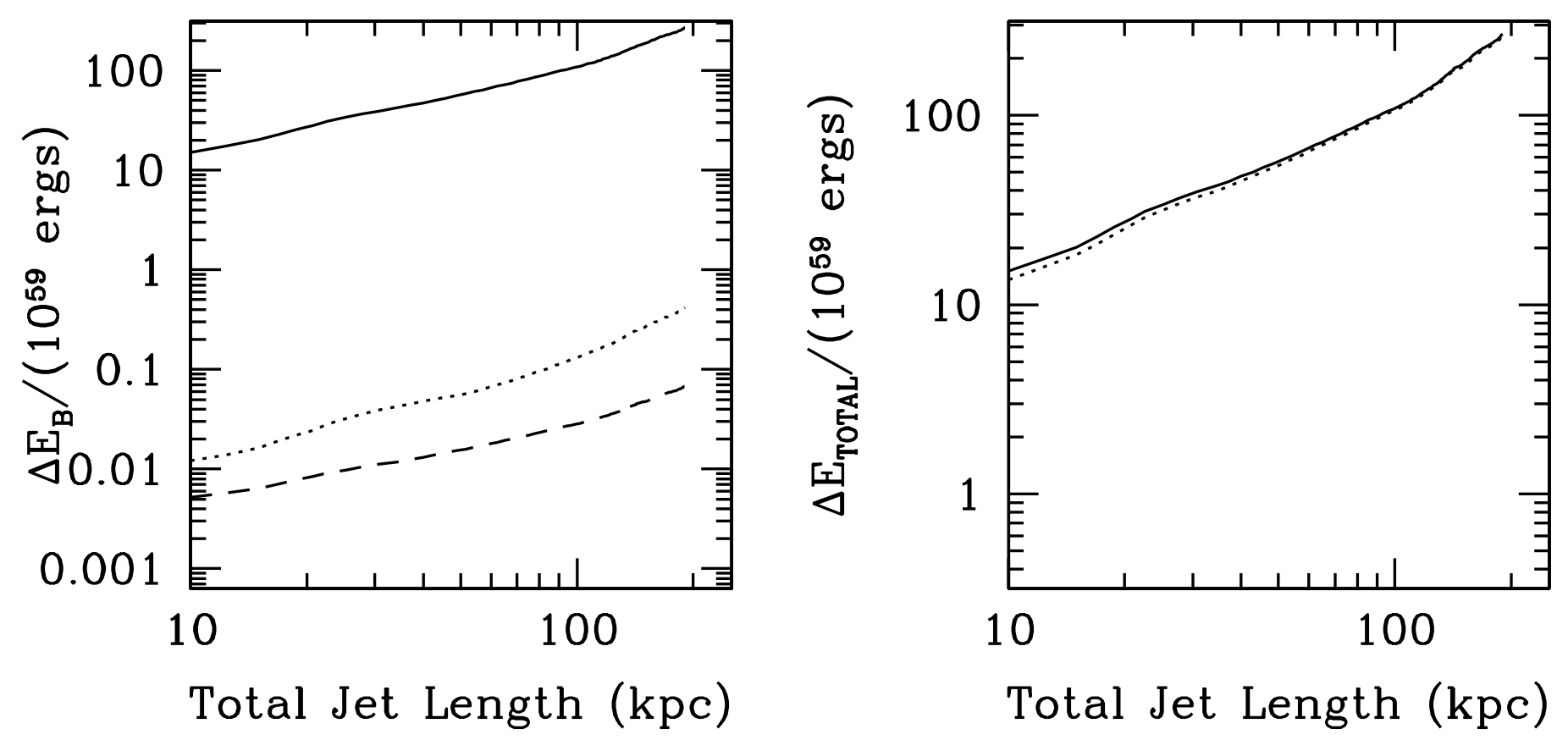

Fig. 7.-Energy flow in the HU-r model. Top left: A comparison of the known added kinetic energy (dashed line) to the measured change (relative to the initial value) in kinetic energy on the grid (dotted line) as they vary with the total jet length. The total (kinetic + thermal + magnetic) inflow energy is shown (solid line) as a reference. Top right: Same, for the thermal energy. Bottom left: Same, for the magnetic energy. Bottom right: Same, for the total energy. [See the electronic edition of the Journal for a color version of this figure.] 

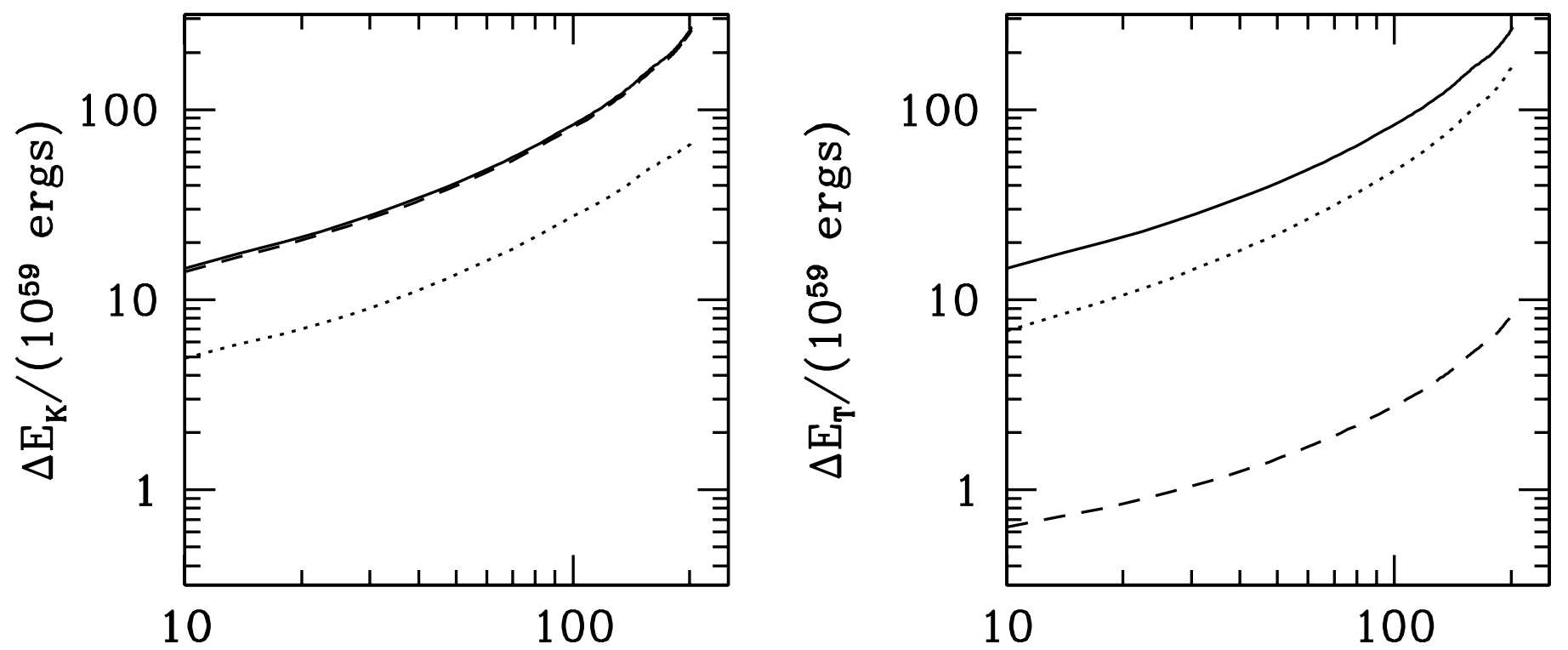

Total Jet Length (kpc)

Total Jet Length (kpc)
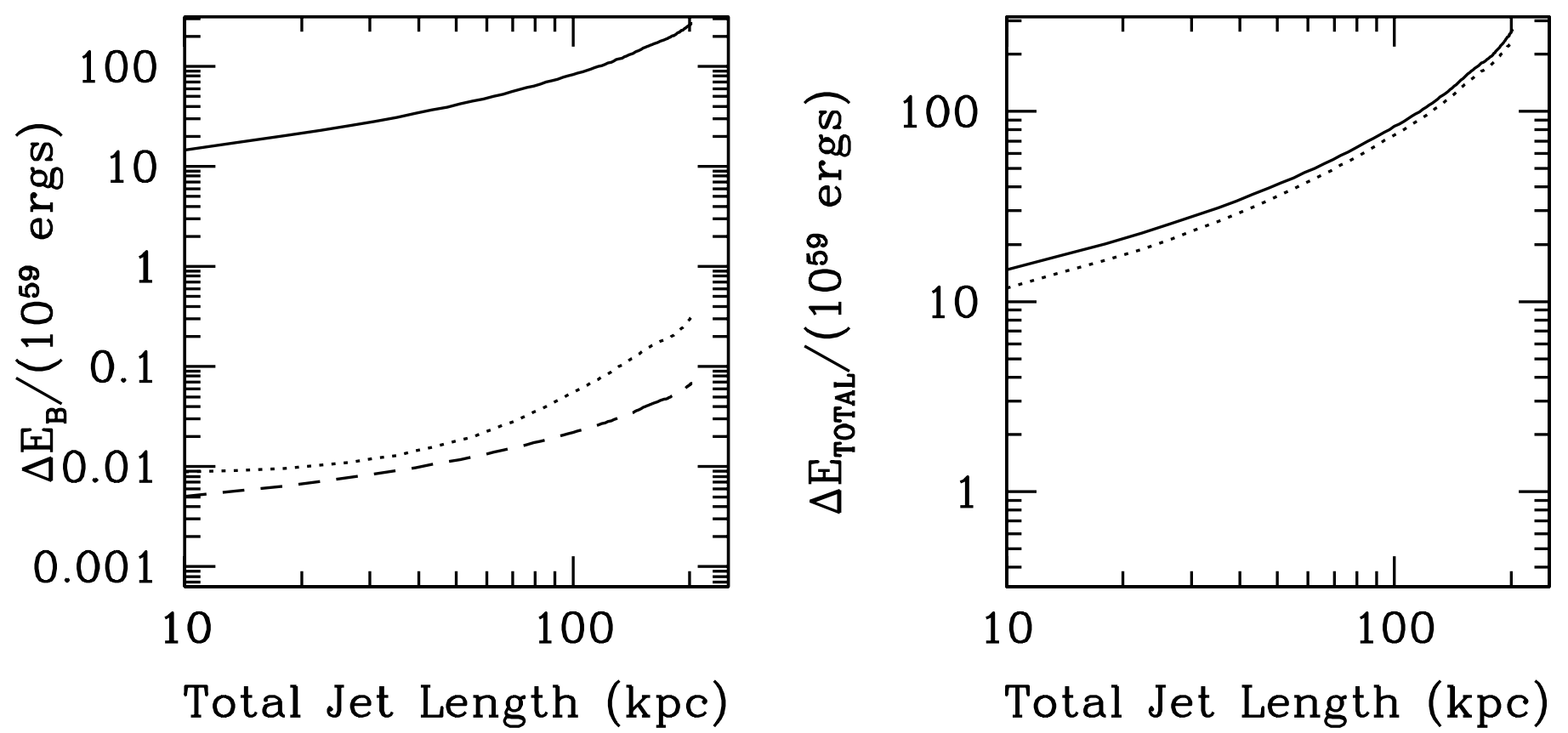

Total Jet Length (kpc)

FIG. 8.-As in Fig. 7, but for the HU model. [See the electronic edition of the Journal for a color version of this figure.] 


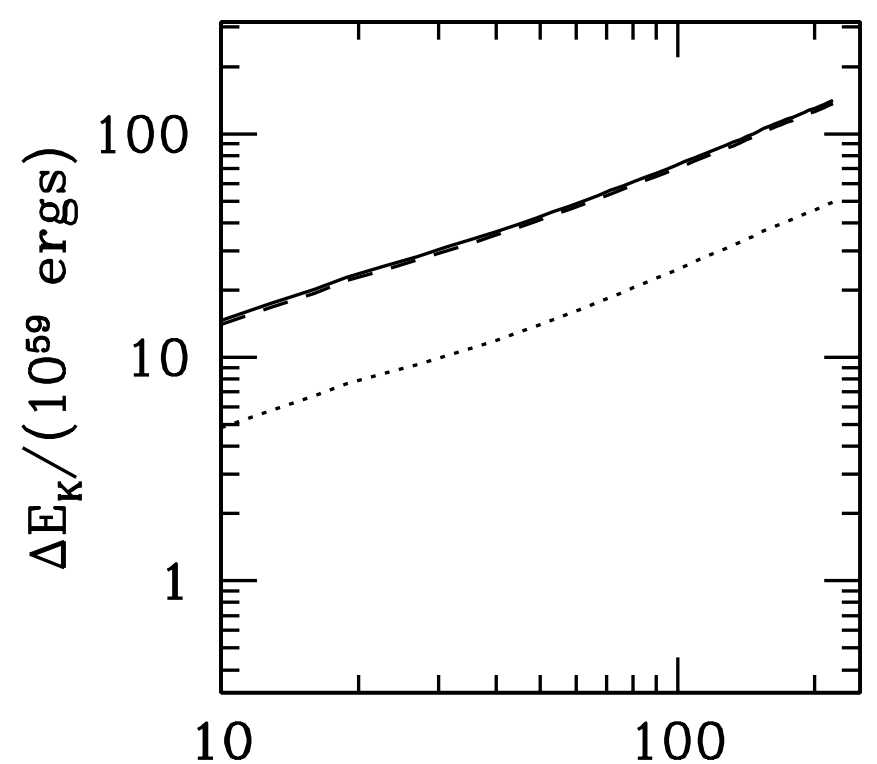

Total Jet Length (kpc)

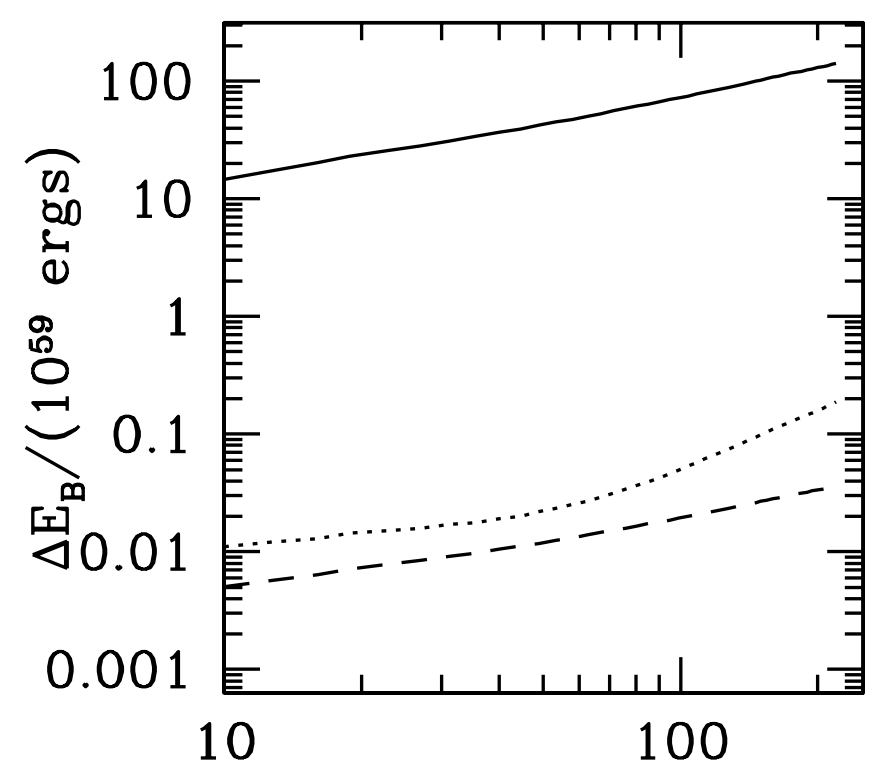

Total Jet Length (kpc)

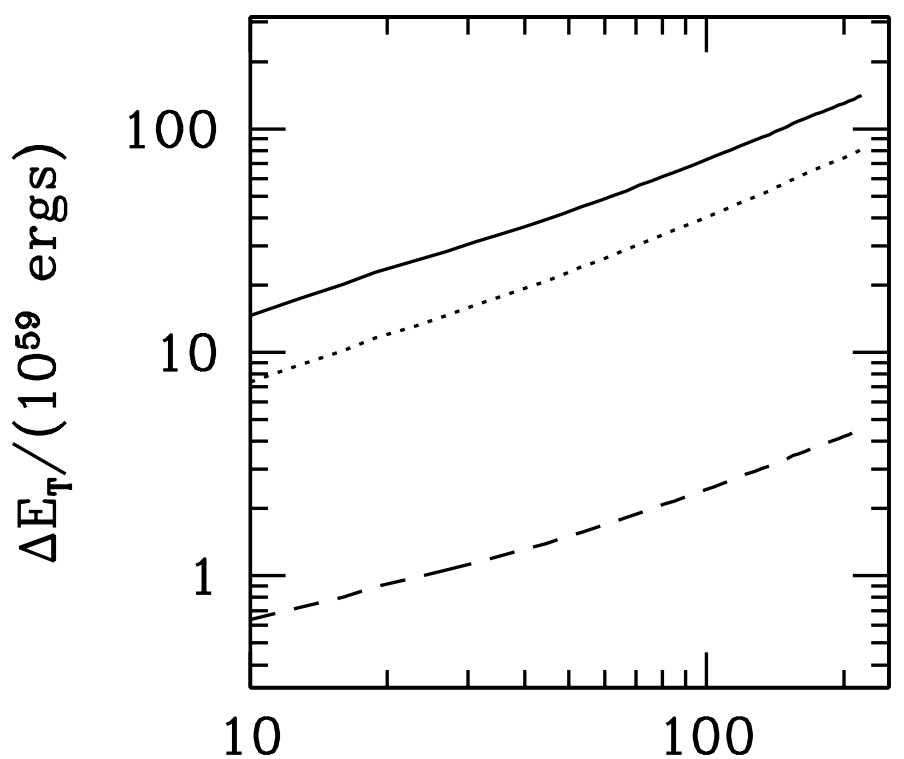

Total Jet Length (kpc)

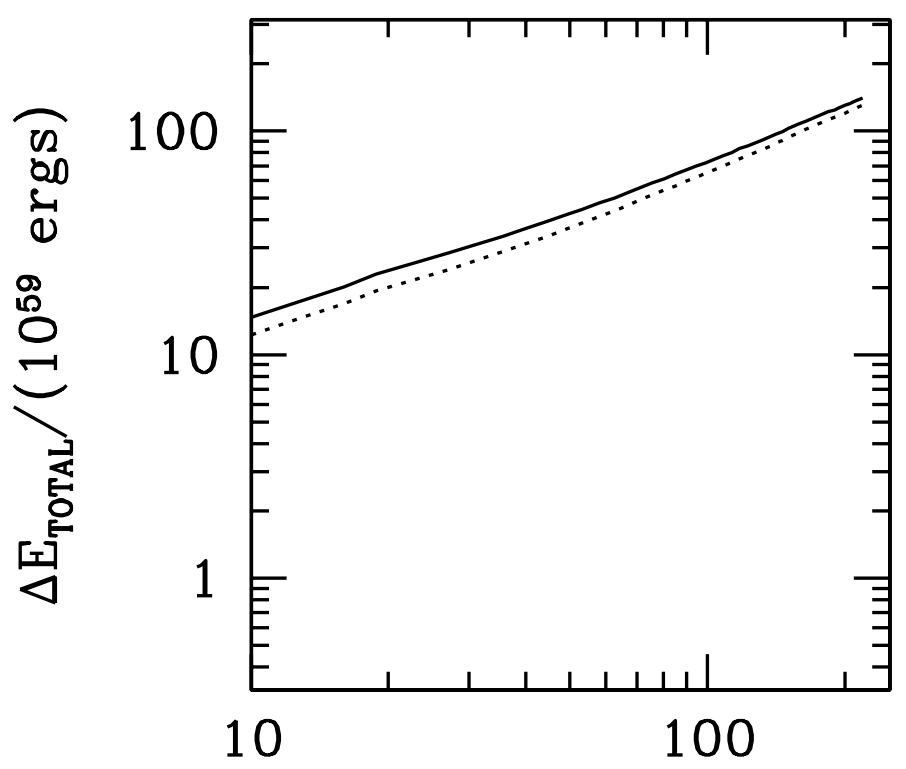

Total Jet Length (kpc)

FIG. 9.-As in Fig. 7, but for the HK model. [See the electronic edition of the Journal for a color version of this figure.] 

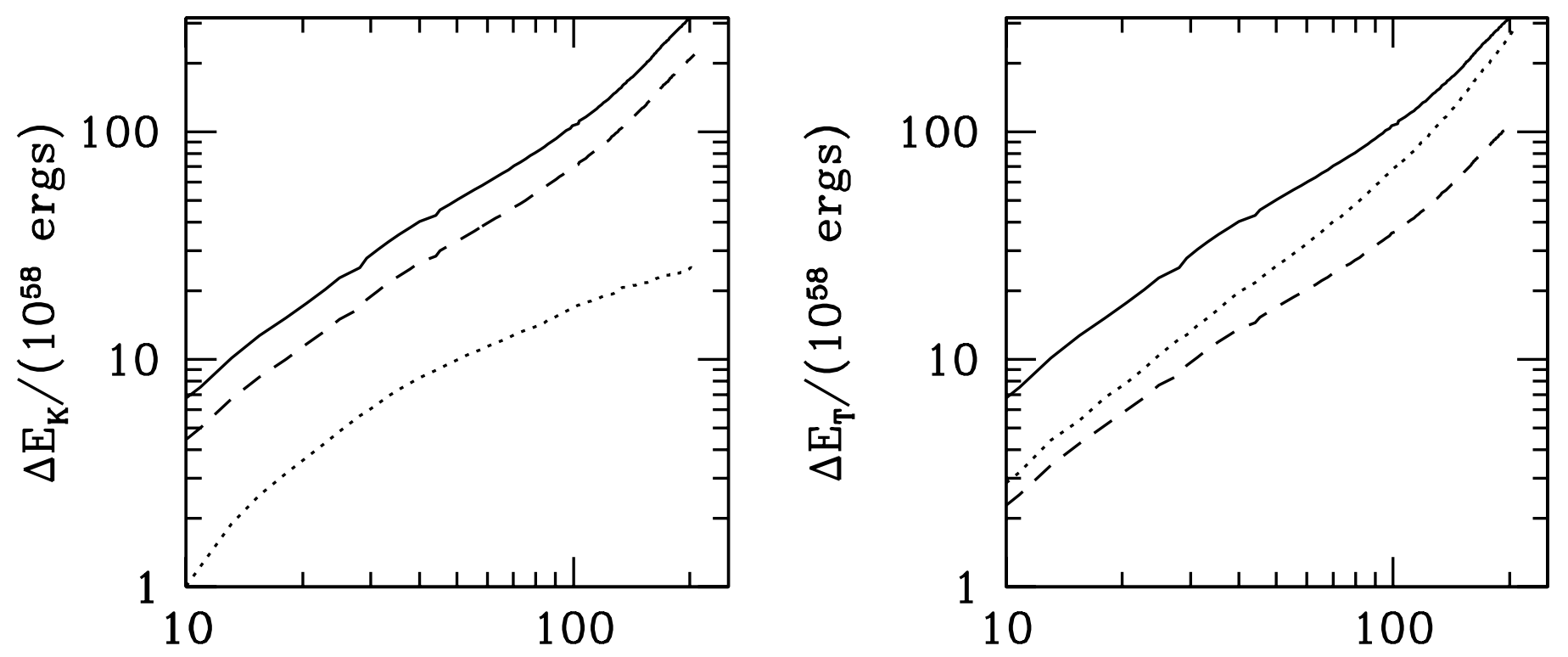

Total Jet Length (kpc)
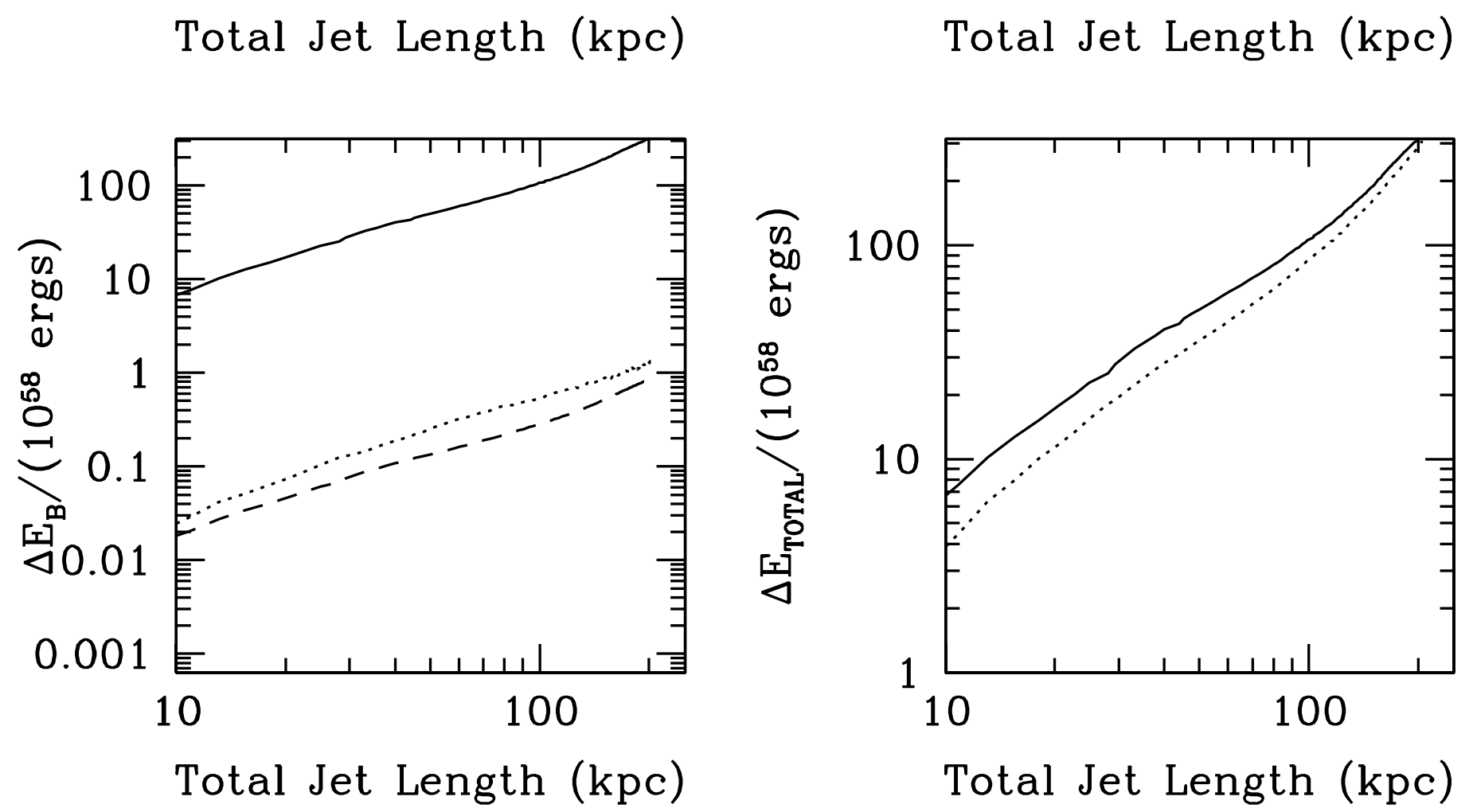

FIG. 10.-As in Fig. 7, but for the LU model. [See the electronic edition of the Journal for a color version of this figure.] 

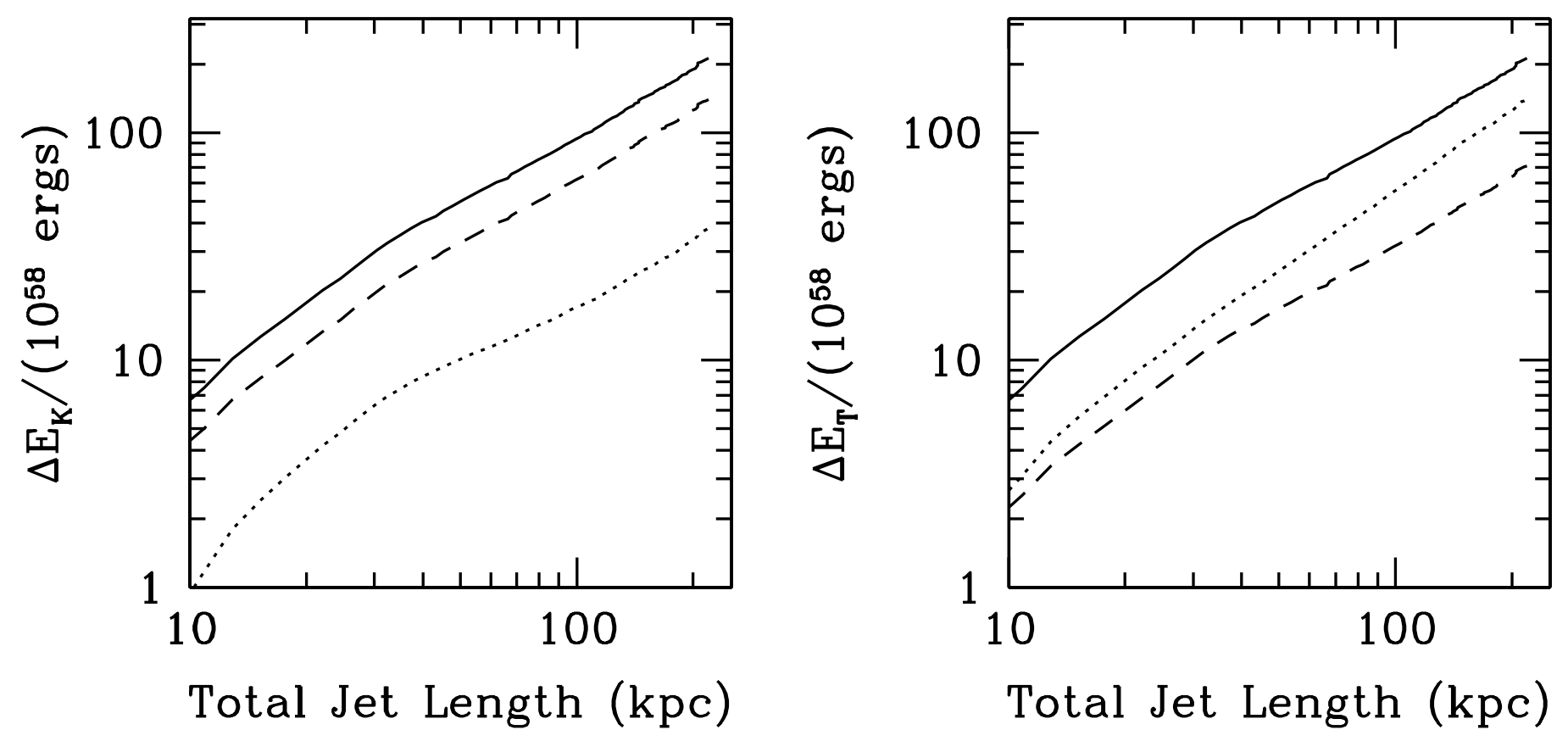

Total Jet Length $(\mathrm{kpc})$

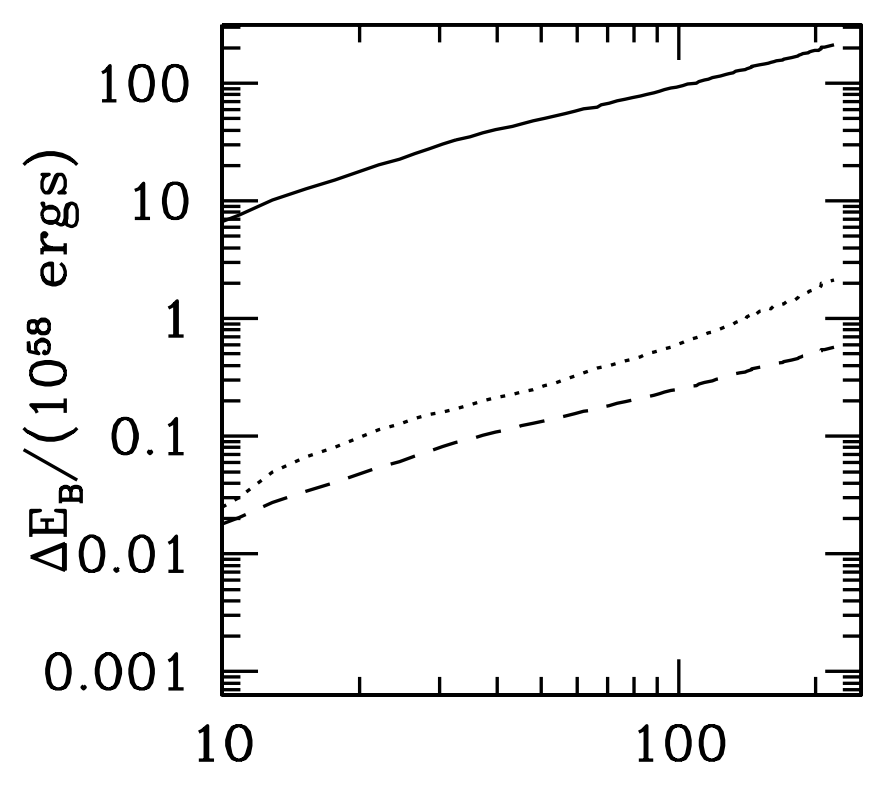

Total Jet Length (kpc)

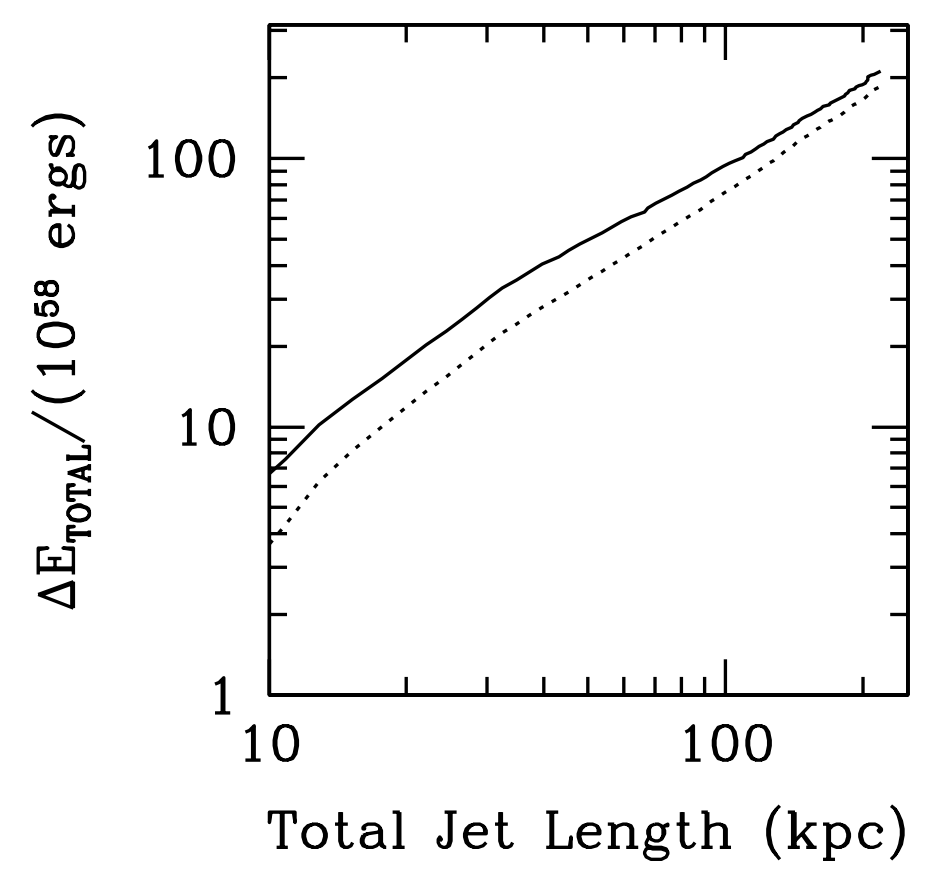

FIG. 11.-As in Fig. 7, but for the LK model. [See the electronic edition of the Journal for a color version of this figure.] 

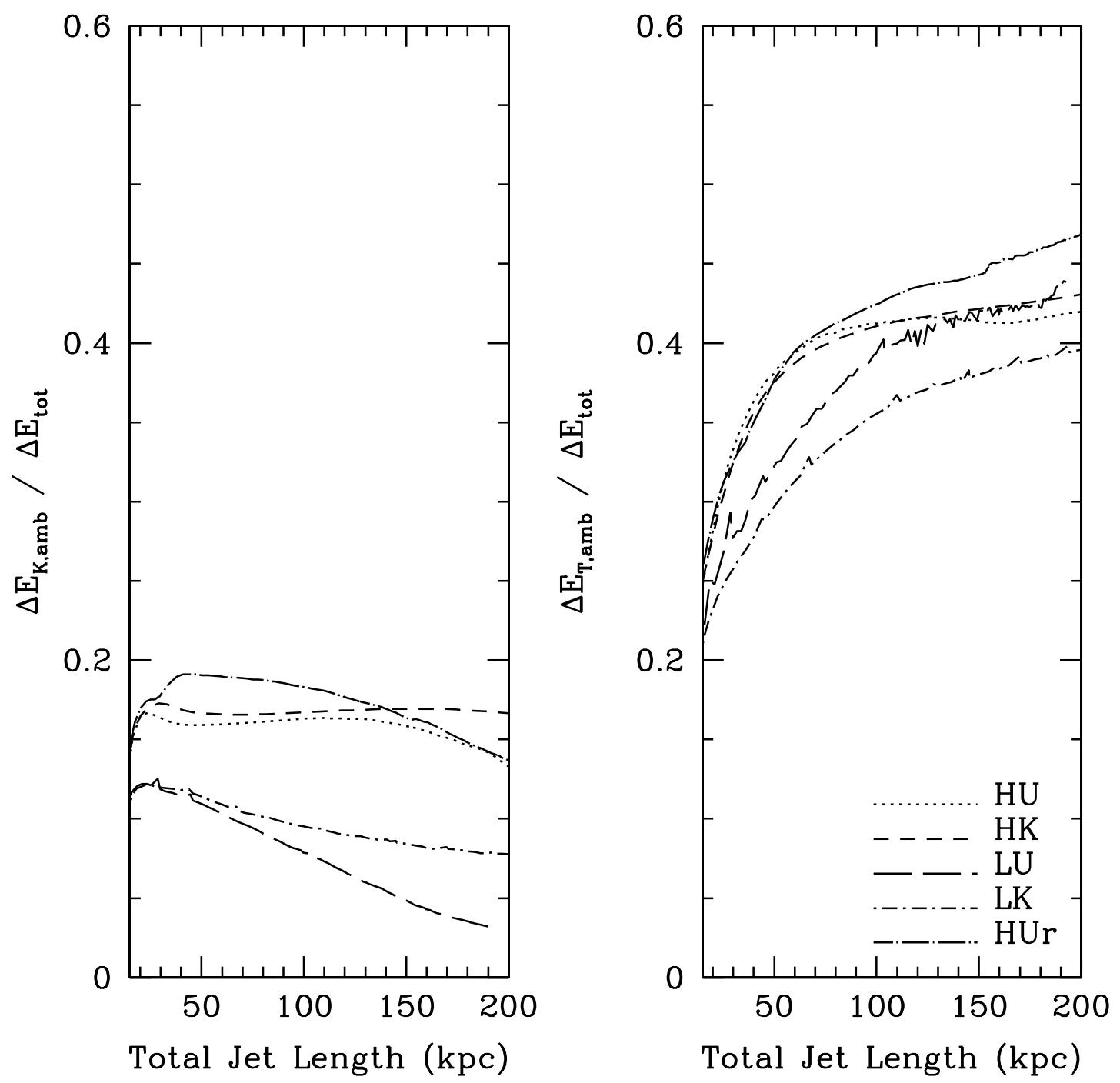

FIG. 12.-Energy deposited in the ambient medium by the jets, as a fraction of total energy added to the grid. The normalized kinetic energy added to the ambient

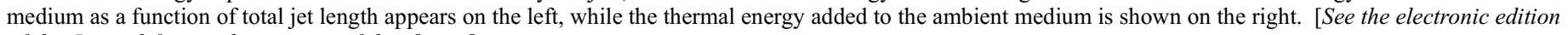
of the Journal for a color version of this figure.]

than ours. However, once again, their dissipation estimates were relatively insensitive to the jet parameters, reinforcing our conclusion above about the general nature of this result. Subsequent to jet switch-off, as one would expect, the bow shock of their inflated jet bubbles continued to dissipate an increasing fraction of the injected energy. In their simulations $\sim 60 \%-70 \%$ of the injected energy was eventually dissipated during that phase, consistent with the classical dissipation behavior of a spherical blast wave (Sedov 1959). MHD simulations of subsonically inflated buoyant bubbles indicate that once the bubble expansion is no longer supersonic, relatively little entropy is added to the ambient medium except through mixing (Jones \& DeYoung 2005).

Examining global energetics now in more detail, we explore how the injected energy is partitioned over the entire grid, including the jet/jet cocoon and the ambient medium. Figures 711 show how the partitioning of energy evolves as a function of total jet length for each model. In each plot, the nominal energy input from the jet as defined by equation (1) is shown as a solid line, for reference. The dashed lines in each plot indicate the nominal energy input of the particular form (e.g., kinetic, thermal, or magnetic). Dotted lines reveal the energy increment of each form actually measured on the grid. The vertical ordering of the dashed and dotted lines indicates the direction of energy conversion (e.g., dashed lines above dotted imply that less energy of that type is measured than was added), while the distance between these lines reflects the amount of energy being transferred to/from a particular form. The lower right plot in each figure provides a comparison between the nominal total energy influx and the measured change in total energy on the grid for that model. As mentioned previously, except for model HU-r, all the models leak some energy across the jet inflow plane, $x=0$, due primarily to escape of cocoon backflow. However, those losses are generally less than $13 \%$ of the inflow energy by the time jet propagation is well established (i.e., once the jet head is well away from the $x=0$ boundary). By that time, backflow velocities near the base of the cocoon as measured in the lab frame are generally only a few percent of the jet speed, while the densities and pressures are much reduced from those in the cocoon head. Consequently, despite the large cross section of the cocoon base, energy fluxes across the boundary are modest. The leakage initially makes up a larger fraction of the inflow energy in the L models, but the asymptotic behaviors of the $\mathrm{L}$ and $\mathrm{H}$ models are similar. In any case, the energy 


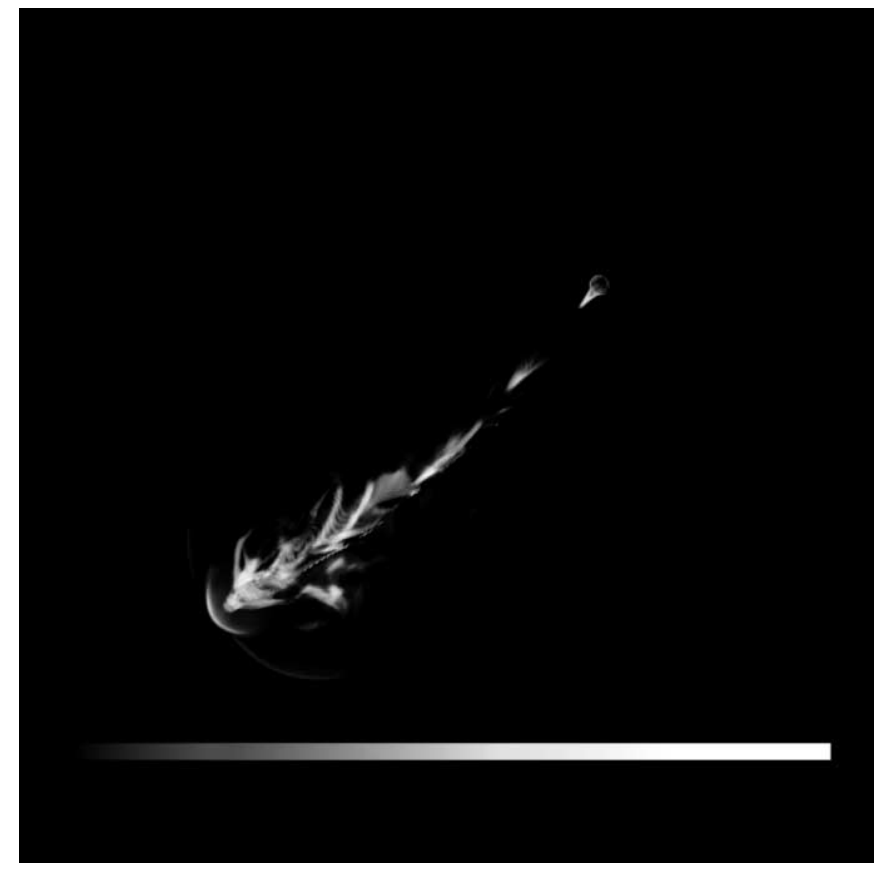

FIG. 13.- - Volume rendering of compression rate for the HU model, after the jet has propagated $\sim 90 \%$ of the total grid length. The color bar runs from low compression rates (dim, dark) to high compression rates (bright, light). [See the electronic edition of the Journal for a color version of this figure and an animation of this quantity as seen from several different angles.]

leakage in the open boundary cases is too small to influence our conclusions.

The global energy flow characteristics of all five simulations are qualitatively similar, especially for cases with the same Mach number. In each case most of the inflowing jet energy is kinetic, of course, because the jets are supersonic and superAlfvénic. The measured kinetic energy increase, however, is always significantly less than the nominal kinetic energy provided by the inflowing jet, indicating that there is always a net conversion of jet kinetic energy into other forms. Gravitational energy is a minor component in all the simulations, and it does not become a significant reservoir. Changes in gravitational energy are generally less than $1 \%$ of the total energy added. As mentioned previously, gravity is included in these simulations only as a method of stabilizing the undisturbed ambient medium, so we ignore it in our discussions. Simulations such as those conducted by Zanni et al. (2005) and Krause (2005) have shown that gravitational energy changes can play a significant role in realistic cluster potentials, but we did not intend to investigate this issue with our models. Although magnetic energy also remains small, it does increase in all the simulations by a substantial factor. Since those changes may be reflected in observable nonthermal emission properties, we include some comments below about magnetic energy variations. Most of the transformed kinetic energy reappears as thermal energy, as our discussion at the start of this section would suggest. There we emphasized that the fractional energy transferred from the jets to ambient thermal energy was similar for all the models during their asymptotic evolution. That similarity applies also to the global transfer of thermal energy.

The relative model independence of the fractional thermal energy transfer is remarkable partly because a large portion of the thermal enhancements is due to shocks, while shock distributions are rather different among the models. To illustrate this last point graphically, Figures 13 and 14 (and the associated

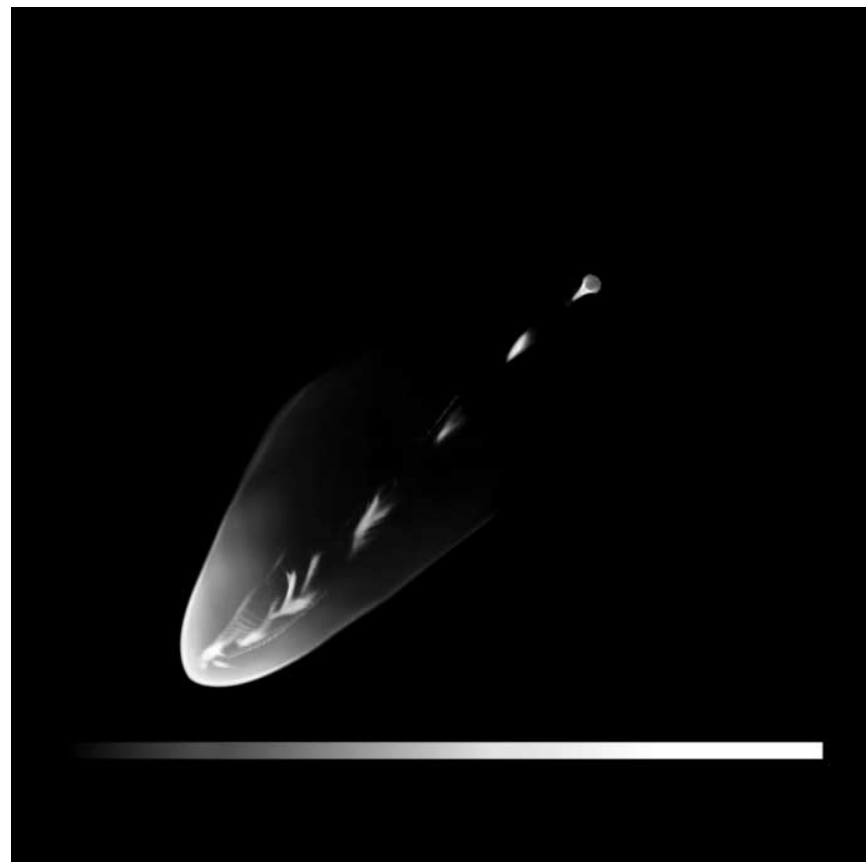

FIG. 14.-As in Fig. 13, but for the HK model. [See the electronic edition of the Journal for a color version of this figure and an animation of this quantity as seen from several different angles.]

animations, available in the electronic version of this paper) show volume renderings of the flow compression rates $(-\nabla \cdot v)$ for the HU and HK runs. The compression rate is a convenient shock tracer that also provides a qualitative indicator of shock strength. In Figure 13 (HU), we see a complicated "shock-web complex" similar to distributions seen in earlier jet simulations in uniform media (e.g., Jones et al. 2001). The shock web is most developed in the jet backflow cocoon, where it is produced by flailing of the jet terminus and especially by collisions between the jet and the wall of its cocoon (Jones et al. 2001; Cox et al. 1991). Some of those shocks are moderately strong, as pointed out previously for similar simulations (Tregillis et al. 2001b, 2004) although many are weak. The same jet-cocoon interactions also generate weaker shocks that penetrate into the ambient medium inside the bow shock. All of these shocks contribute to dissipation of jet kinetic energy. In contrast to the $\mathrm{HU}$ case, the HK jet illustrated in Figure 14 exhibits a noticeably more stable propagation, and only near the very end of the simulation does it begin to develop an evident shock web near its head. This difference in jet behaviors in uniform and stratified media derives from the strong deceleration of the jet head that develops in uniform media, in contrast to an almost constant extension of the jet in the $\mathrm{K}$ model environments. The former leads to a much stronger back-reaction on the jet tip from the ambient medium. The similar fractions of jet energy that are irreversibly dissipated under these different circumstances emphasizes that in either case the jet thrust must be transferred to its surroundings, which roughly defines the pressure surrounding the jet as it propagates. That pressure is produced largely through shocks, which can be simple and strong or complex but individually weak, so long as their accumulated effect is the same.

Just as we have seen for the thermal energy generation, Figures 7-11 show that the total magnetic energy increases in each model exceed the magnetic energy represented by the jet Poynting flux. Early in the simulations this excess is typically a factor of $\sim 2$ in each case. Except for the LU simulation, the 


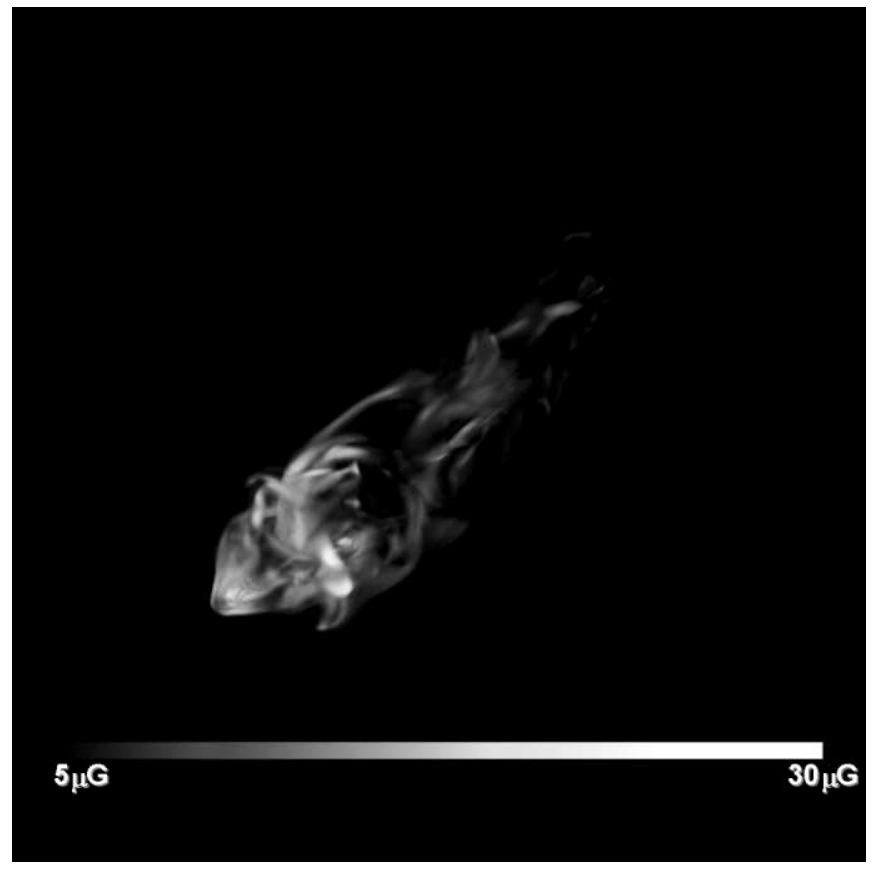

FIG. 15.-Volume renderings of the log of magnetic field strength for the HU model, after the jet has propagated $\sim 90 \%$ of the total grid length. In this case, the bow shock has been removed to enhance visualization of the jet and cocoon. [See the electronic edition of the Journal for a color version of this figure and an animation of this quantity as seen from several different angles.]

relative magnetic energy enhancement increases to at least a factor of 5 by the end of each simulation. Just as they were for shocks, the distributions of magnetic fields are rather different, despite the similar energetics. This is illustrated by the contrasting magnetic field distributions in the HU and HK simulations shown in Figures 15 and 16 (and the associated animations, available in the electronic version of this paper). Those differences derive from the same dynamical distinctions mentioned in reference to shocks. Most of the magnetic field enhancements can be traced to compression events in the flows (especially shocks), followed by flux stretching.

We end this section by pointing out one important detail that results from the differences in propagation histories of the $\mathrm{U}$ and $\mathrm{K}$ simulations for a given power class ( $\mathrm{H}$ or $\mathrm{L}$ ). In particular, since jets propagating through a stratified atmosphere exhibit much less deceleration, their lengths are greater for a given age. Consequently, if we consider the total energy budget of jets of a given length, the energy deposited by the $U$ jets will be substantially greater. For example, by the time the jets have reached $90 \%$ of the grid length, the HU jet has advected onto the grid twice as much energy as the HK, while the LU has advected 1.75 times as much energy as the LK. For both sets of Mach numbers, this difference is appreciable and is noticeable in the backflow, especially in the high Mach number jets. Figures 2 and 3, for example, show that there is more material flowing opposite to the direction of jet propagation in the HU case than in the HK, which is indicative of a greater amount of kinetic energy in the system at a given jet length.

\subsection{Dynamics and Morphology}

We now explore some basics of the dynamical evolution of our simulations, with emphasis on how the cocoon length and volume depend on the ambient density structure. We pay particular attention to asymptotic behaviors, comparing them to simple models and previous two-dimensional simulations. The

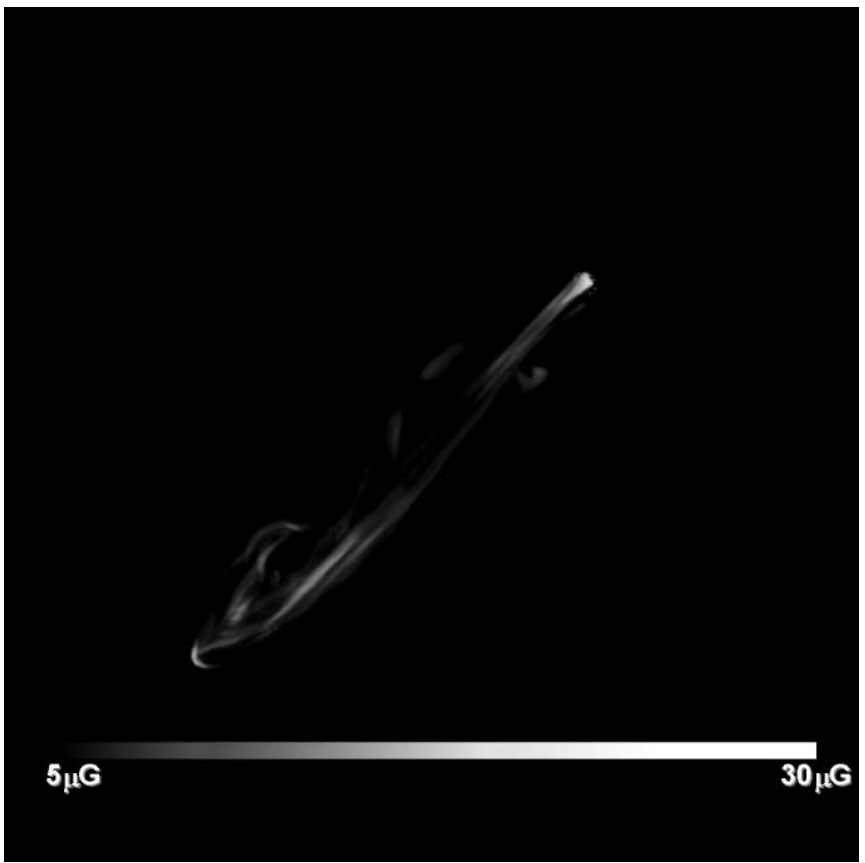

FIG. 16.-As in Fig. 15, but for the HK model. [See the electronic edition of the Journal for a color version of this figure and an animation of this quantity as seen from several different angles.]

most basic dynamical features of jet evolution are the length, $l$, and the speed of the jet head, $v$, as functions of time. The simplest approach to modeling these uses dimensional analysis based on the energy deposited by the jet; i.e., one applies an extension of the familiar Sedov-Taylor analysis (e.g., Falle 1991; Heinz et al. 1998; Zanni et al. 2005). Our simulated jets are steady, and we found above that a relatively constant fraction of the jet power is transferred into thermal energy in the cocoon and the shocked ambient medium, at least asymptotically. We assume, therefore, that the energy being stored increases linearly with time. Similarly, the ratio of energy in the shocked ambient medium and the cocoon becomes relatively steady. The more directly observable of these volumes is likely to be the cocoon, since it corresponds to the radio lobe of a radio galaxy. Thus, we use that volume for our dimensional analysis and assume that the energy content of the cocoon is proportional to the energy shared with swept-up ambient matter. To analytically estimate the mass swept up by the cocoon, one must make some assumption about the cocoon volume as a function of length. It is common to assume a sphere, although to obtain a scaling it is really necessary only to assume a volume form, $V(l)$. A convenient generalization of the spherical assumption is $V(l) \propto l^{1+\alpha}$. Any homologous structure, including the sphere, would correspond to $\alpha=2$. Assuming that the ambient density follows a simple power law of the form $\rho=\rho_{0}\left(x / x_{c}\right)^{-\kappa}$, the similarity length then scales as $l \propto t^{3 /(3+\alpha-\kappa)}$, while the similarity speed follows the form $v \propto t^{(\kappa-\alpha) /(3+\alpha-\kappa)}$, or $v \propto l^{(\kappa-\alpha) / 3}$.

Figure 17 plots the measured cocoon volumes for each of our simulated flows as functions of jet length, while Figure 18 shows the measured length evolution and the measured head propagation speed for each case. The cocoon volume corresponds to material with $C_{j} \geq 0.90$. Although this method of accounting includes the jet in the cocoon volume estimate, the volume of the jet itself is always less than $10 \%$ that of the cocoon, and this fraction decreases in time after the flow is well established. It is worth noting that the characteristic cocoon widths, as estimated by assuming a cylindrical cocoon of the 


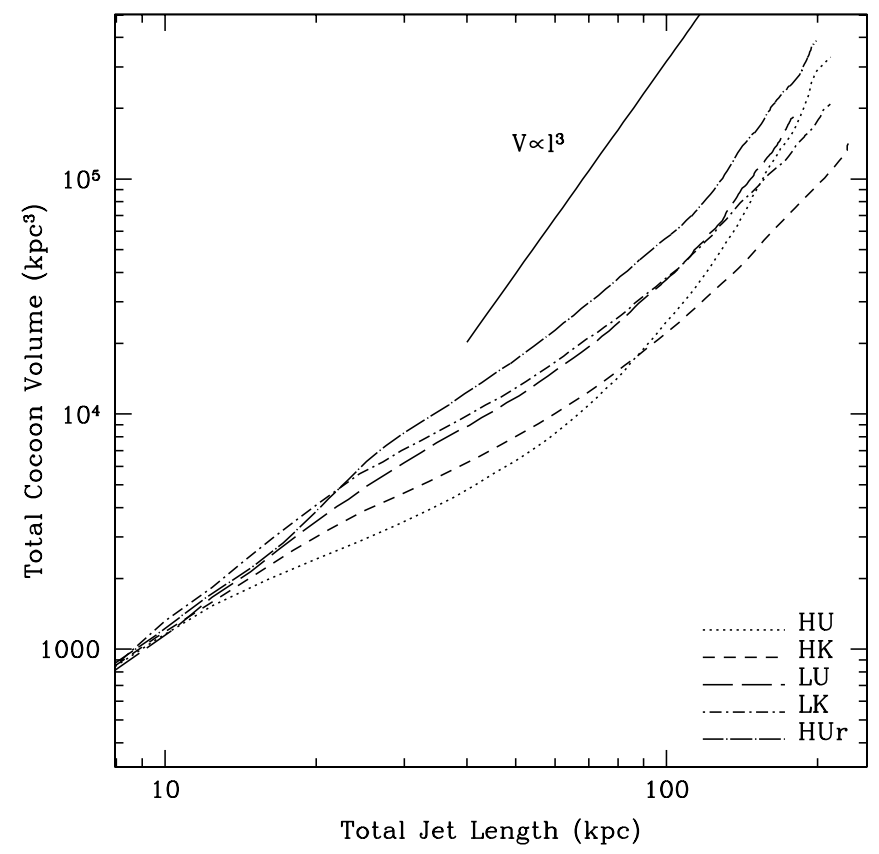

FIG. 17.-Cocoon volumes, plotted as a function of total jet length for all models. The solid line represents the slope for the simple scaling-law expectation. [See the electronic edition of the Journal for a color version of this figure.]

observed volume and length, are comparable in magnitude to the projected amplitude of the jet wobble, differing by roughly a factor of 2 at most. We did not explore the dependence of this characteristic cocoon width on jet opening angle, but we do note that a cocoon volume dominated exclusively by the initial jet wobble would produce $V \propto l^{3}$ at all times, which is not seen.

The early cocoon evolution, when $l \lesssim 20-30 \mathrm{kpc}$, seems to follow a form close to $V \propto l^{3 / 2}$, or $\alpha=1 / 2$, for all the simulations. This interval corresponds roughly to the times before the jets are recollimated after entering the grid. All the jets are still well inside uniform ambient media on those scales, so the anticipated similarity forms would be $l \propto t^{6 / 7}, v \propto l^{-1 / 6}$. The early velocity and especially the length evolution poorly approximate this, showing instead a flatter relation. This breakdown of self-similar evolution should not be too surprising in the initial dynamical stages, partly on account of residual startup behaviors. The energy partitioning is not steady during this interval. This is also the regime where the simulations with open boundaries along the $x=0$ plane suffer the most significant energy losses. We note, however, that the HU-r simulation, which does not suffer such losses, is not a particularly closer match to the self-similar form. We should keep in mind, as well, that the flow pattern is still only a few jet radii in size (recall that $\left.r_{j}=2 \mathrm{kpc}\right)$.

On intermediate scales, roughly $20-30 \mathrm{kpc} \lesssim l \lesssim 70 \mathrm{kpc}$, all of the volume behaviors are consistent with approximately $\alpha \sim 1 / 3$. This regime corresponds to the intervals between jet recollimation and when the $\mathrm{K}$ model jets begin to emerge from the core density region. In this regime the volume scaling shows that the length of the jet extends much more rapidly than the width of the cocoon. Looking again at Figure 12, we see that the thermalization of jet power still has not reached its asymptotic level. The cocoon is not being driven strongly laterally by internal pressure. Again setting $\kappa=0$ in this domain, the anticipated similarity length and velocity scalings would be $l \propto t^{9 / 10}$, $v \propto l^{-1 / 9}$. Here we find (Fig. 18) a much better match with the measured length and velocity behaviors for all of the jets.
Eventually, the volume scalings steepen into something approximating $V \propto l^{3}(\alpha=2)$, but only after the jet lengths exceed about $50 r_{j}(100 \mathrm{kpc})$. This transition corresponds to the cocoons becoming strongly laterally driven by internal pressure. A fiducial line with slope 3 is included in Figure 17 for comparison. Since the K model jets extend into the strongly stratified medium by that time, two different scaling relations are expected. For the $\mathrm{U}$ media, with $\kappa=0$, the forms are $l \propto t^{3 / 5}, v \propto l^{-2 / 3}$. For the $\mathrm{K}$ models, $\kappa=2$, so we expect $l \propto t$, $v=$ constant. Looking at Figure 18 and using the fiducial lines provided, we can see a fairly good correspondence with the measured behaviors once the jet lengths exceed $\sim 100 \mathrm{kpc}$. We conclude, therefore, that asymptotically our jets and their cocoons do evolve consistent with simple self-similar forms. These behaviors are roughly consistent with those found from two-dimensional axisymmetric models and similar dimensional arguments by Carvalho \& O'Dea (2002a, 2002b). We note that a more realistic model of cluster gravity and a full threedimensional spherically symmetric King profile has the potential to affect evolved flow structures by squeezing the cocoon, as discussed by Alexander (2002) and seen in simulations by Krause (2005).

We add that Carvalho \& O'Dea (2002a, 2002b) also explicitly modeled the evolution of the cocoon volume based on their twodimensional axisymmetric simulations. Their two-dimensional results for $\mathrm{K}$ model atmospheres are comparable to our $\mathrm{K}$ model atmospheres in three dimensions. The scaling expectations, where in all cases $\alpha \approx 2$, are thus consistent with both the twodimensional results and our asymptotic $\mathrm{K}$ models. The uniform atmospheres in Carvalho \& O'Dea (2002a) produce $\alpha \approx 2 / 3-$ $7 / 3$ for the HU parameters and $\alpha \approx 5 / 4-3 / 2$ for the LU parameters. For the HU run, this range includes our value of $\alpha \approx 2$, but our LU cocoon evolves slightly outside of the range of the twodimensional version. As in the case of jet length, the evolution of our LU model more closely resembles that of our HU model than that of the two-dimensional analog, indicating that, for our range of Mach numbers, three-dimensional morphology evolution is not strongly influenced by Mach number. Although the flows may have morphologies distinct from one another, the evolved jet lengths and cocoon sizes are all adequately described by simple scaling relations listed above.

To conclude this section, we briefly compare the dynamical evolution of the HU and HU-r simulations. Recall that the only difference between these was the character of the boundary along the jet inflow plane, $x=0$. The HU simulation left this boundary open outside the jet orifice, while the HU-r simulation applied reflection conditions outside the jet orifice, in order to prevent any energy or mass loss there. As Figures 17 and 18 illustrate, the asymptotic volumes, lengths and head speeds of these two simulations are very similar, so that the choice of boundary condition has not played a major role in their longterm evolution. The HU jet is slightly longer at a given time, while at the end of the simulations the HU-r cocoon volume is slightly larger for a given length. At intermediate times and lengths, however, the evolutionary behaviors are more obviously distinct. In particular, the HU-r cocoon inflates significantly faster, while the HU head advances significantly faster. Comparing Figure 7 and Figure 8 reveals that the thermal energy enhancement is still significantly greater in the HU-r simulation during this interval. The open boundary in the HU simulation has reduced the accumulated cocoon energy near the inflow plane, reducing the cocoon inflation there. As the jet length increases, however, pressure gradients close to the inflow plane become smaller, so that less and less of the jet 

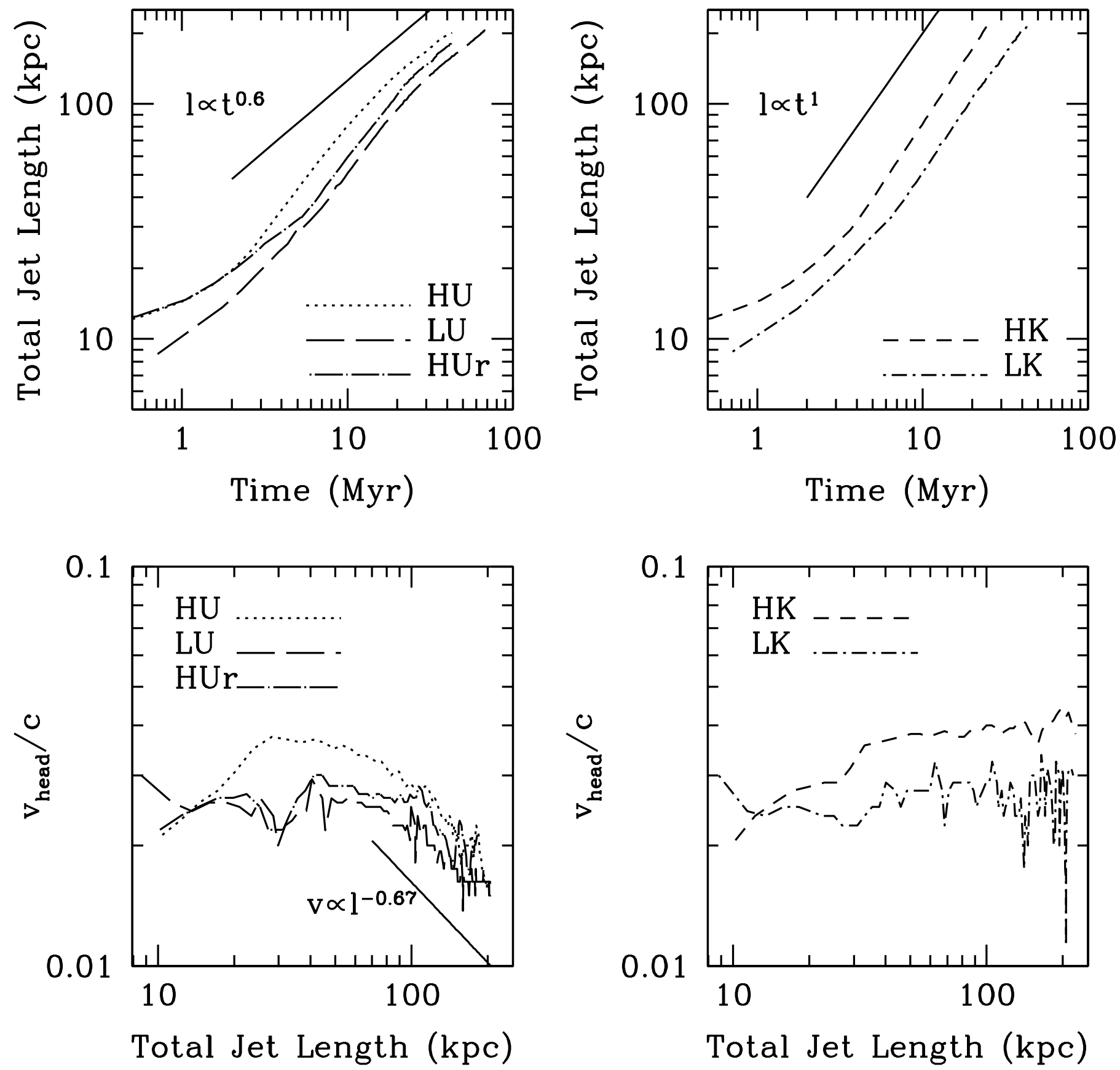

FIG. 18. - Jet evolution, showing jet length as a function of time for the uniform models (top left) and the King models (top right), and jet head speed as a function of jet length for the uniform models (bottom left) and the King models (bottom right). The solid lines represent the slopes for the simple scaling-law expectations. [See the electronic edition of the Journal for a color version of this figure.]

power escapes across this plane, and the boundary plays a smaller and smaller role in the evolution of the jet and its cocoon

\section{CONCLUSIONS AND ASTROPHYSICAL IMPLICATIONS}

We have conducted a series of five simulations to explore the influence of Mach number and external environment on the large-scale propagation of steady, light MHD jets. We explored the flow of energy in our simulated systems, tracking both the global partitioning of energy in its various forms and the spatial distribution of energy as the jets evolved, seeking to determine the extent to which energy is transferred from these jets to their surroundings. We further examined the morphologies and dy- namics of our simulated jets and compared our data to simple dimensional scaling expectations and the results of analogous simulations in two dimensions.

Our simulations suggest that energy transfer from evolved jets to their environments is remarkably efficient. Approximately half of the energy flux of an evolved jet enters the ambient medium, and roughly half of this (one-quarter of the total flux) goes directly toward dissipative heating of the ambient medium. This trend is present for all of our models, with various Mach numbers and ambient medium structures, suggesting that energy transfer and dissipation is not strongly dependent on the exact nature of the flow. This result is important because high rates of energy flux into the ambient 
medium make AGNs good candidates for reheating of their ICM environments.

We also find that simple energy-based similarity scaling laws reasonably describe the asymptotic time evolution of jet length and cocoon size in our simulations, independent of our model parameters. This lends further support to the employment of these simple relations in estimating radio lobe energy densities and estimating flow properties. Although some observed structures may depend on the unique history of a particular object, our simulations suggest that very basic size parameters are welldescribed by simple scaling laws.

To reiterate, the most important results from our work are:

1. Energy transfer to the ambient medium is efficient for all of our simulations, with approximately half of the inflowing jet energy becoming thermal energy in the ambient medium while the jet propagates. Roughly half of this energy is added irreversibly through dissipation, mostly at shocks. This characteristic behavior is independent of our models, suggesting that active jet reheating of the ICM can be efficient under a variety of physical circumstances, as is the case in two-dimensional simulations. Furthermore, this trend is independent of the differences in shock and magnetic field structure that occur in our various models. This suggests that real radio galaxies may transfer energy equally efficiently to the ambient medium, despite having a variety of brightness distributions.
2. Dynamically, our jets asymptotically resemble simple dimensional scaling expectations and previous results in two dimensions. This should suggest that supersonic jets are well described by simple models for their behavior, and that it is reasonable to use such scalings as starting points for models of jet luminosity and morphology evolution.

3. Since jets propagate to greater lengths in a given time through stratified media than through uniform media, the jet lobe energy content from jets offixed power and length depends significantly on the density profiles of their environments. For a given Mach number and jet length, this has the effect of introducing more energy into uniform environments than stratified atmospheres. In addition, as our jets are disrupted, they convert a larger fraction of their inflow to thermal energy. This implies that stalled jets in uniform atmospheres may eventually become even more efficient at generating thermal energy, most of which will eventually enter the ambient medium.

This work by S. M. O. and T. W. J. was supported by the NSF grant AST 03-07600 and by the Minnesota Supercomputing Institute. The work by D. R. was supported by the KOSEF grant R01-2004-000-10005-0. We also wish to thank Brian Cornell for his work in developing some of our visualization tools.
Alexander, P. 2000, MNRAS, 319, 8 2002, MNRAS, 335, 610

Blanton, E. L., Sarazin, C. L., \& McNamara, B. R. 2003, ApJ, 585, 227

Böhringer, H., Matsushita, K., Churazov, E., Ikebe, Y., \& Chen, Y. 2002, A\&A, 382,804

Brüggen, M., Kaiser, C., Churazov, E., \& Ensslin, T. 2002, MNRAS, 331, 545

Carvalho, J. C., \& O’Dea, C. P. 2002a, ApJS, 141, 337 2002b, ApJS, 141, 371

Churazov, E., Forman, W., Jones, C., \& Böhringer, H. 2003, ApJ, 590, 225

Cioffi, D. F., \& Blondin, J. M. 1992, ApJ, 392, 458

Cox, C. I., Gull, S. F., \& Scheuer, P. A. G. 1991, MNRAS, 252, 558

Fabian, A. C., et al. 2003, MNRAS, 344, L43

Falle, S. A. E. G. 1991, MNRAS, 250, 581

Hardee, P. E., \& Clarke, D. A. 1992, ApJ, 400, L9

Hardee, P. E., White, R. E. III, Norman, M. L., Cooper, M. A., \& Clarke, D. A. 1992, ApJ, 387, 460

Heinz, S., Reynolds, C., \& Begelman, M. C. 1998, ApJ, 501, 126

Hooda, J. S., \& Wiita, P. J. 1996, ApJ, 470, 211

Jones, T. W., \& DeYoung, D. S. 2005, ApJ, 624, 586

Jones, T. W., Ryu, D., \& Engel, A. 1999, ApJ, 512, 105

Jones, T. W., Tregillis, I. L., \& Ryu, D. 2001 in ASP Conf. Ser. 250, Particles and Fields in Radio Galaxies, ed. R. A. Laing \& K. M. Blundell (San Francisco: ASP), 324

\section{REFERENCES}

Kaiser, C. R., \& Alexander, P. 1997, MNRAS, 286, 215

King, I. 1962, AJ, 67, 471

Komissarov, S. S., \& Falle, S. A. E. G. 1997, MNRAS, 288, 833 1998, MNRAS, 297, 1087

Krause, M. 2003, A\&A, 398, 113

2005, A\&A, 431, 45

Norman, M. L. 1996, in ASP Conf. Ser. 100, Energy Transport in Radio Galaxies and Quasars, ed. P. E. Hardee, A. H. Bridle, \& J. A. Zensus (San Francisco: ASP), 319

Norman, M. L., Winkler, K.-H. A., Smarr, L., \& Smith, M. D. 1982, A\&A, 113, 285

Robinson, K. et al. 2004, ApJ, 601, 621

Ryu, D., \& Jones, T. W. 1995, ApJ, 442, 228

Ryu, D., Miniati, F., Jones, T. W., \& Frank, A. 1998, ApJ, 509, 244

Sedov, L. I. 1959, Similarity and Dimensional Methods in Mechanics (London: Academic Press)

Tregillis, I. L., Jones, T. W., \& Ryu, D. 2001a in ASP Conf. Ser. 250, Particles and Fields in Radio Galaxies, ed. R. A. Laing \& K. M. Blundell (San Francisco: ASP), 336

2001b, ApJ, 557, 475

2004, ApJ, 601, 778

Zanni, C., Murante, G., Bodo, G., Massaglia, S., Rossi, P., \& Ferrari, A. 2005, A\&A, 429, 399 